\title{
A Novel Ship Detector Based on the Generalized-Likelihood Ratio Test for SAR Imagery
}

\author{
Pasquale Iervolino, Member, IEEE, and Raffaella Guida, Member, IEEE
}

\begin{abstract}
Ship detection with synthetic aperture radar (SAR) images, acquired at different working frequencies, is presented in this paper where a novel technique is proposed based on the generalized-likelihood ratio test (GLRT). Suitable electromagnetic models for both the sea clutter and the signal backscattered from the ship are considered in the new technique in order to improve the detector performance. The GLRT is compared to the traditional constant false alarm rate (CFAR) algorithm through Monte-Carlo simulations in terms of receiver operating characteristic (ROC) curves and computational load at different bands (S-, C-, and X-). Performances are also compared through simulations with different orbital and scene parameters at fixed values of band and polarization. The GLRT is then applied to real datasets acquired from different sensors (TerraSAR-X, Sentinel-1, and Airbus airborne demonstrator) operating at different bands (S-, C-, and $\mathrm{X}$-). An analysis of the target-to-clutter ratio (TCR) is then performed and detection outcomes are compared with an automatic identification system data when available. Simulations show that the GLRT presents better ROCs than those obtained through the CFAR algorithm. On the other side, results on real SAR images demonstrate that the proposed approach greatly improves the TCR (between 22 and $32 \mathrm{~dB}$ on average), but its computational time is 1.5 times slower when compared to the CFAR algorithm.
\end{abstract}

Index Terms-Maximum likelihood estimation, radar crosssections, radar detection, ship detection, synthetic aperture radar.

\section{INTRODUCTION}

$\mathbf{T}$ HE request for maritime security and safety applications is increased in the recent years due to the growing interest in maritime surveillance. In this scenario, one of the operational applications is the ship detection [13]-[24], with main requirements to be: high probability of detection (PD) (ideally 1 ), low probability of false alarm (PFA) (ideally 0), accurate geolocation, ship identification, and ability to operate in all weather and light conditions. Unfortunately, there is no single mean today able to address all the above requirements at the same time. For this reason, novel monitoring techniques, which merge multisources data, are being developed.

The European Maritime Safety Agency, for example, has developed tools for both oil spills identification (CleanSeaNet) [1] and ship detection (SafeSeaNet) [2]. These tools rely also on synthetic aperture radar (SAR) images. SARs are very successful in the detection of noncooperative ships and the tracking of small vessels without automatic identification systems (AIS) on board [3], being able to offer complementary capabilities to AIS and long-range identification and tracking systems [4]. CleanSeaNet and SafeSeaNet services aim to strengthen operational responses to accidental and deliberate discharges from ships and cargos assisting UE States to locate and identify polluters in areas under their jurisdiction [1], [2]. In particular, the services employ three polar orbiting SAR satellites: the Canadian RADARSAT-2 [5], the Italian COSMO-SkyMed [6], and the European Union's ENVISAT [7] (operative until May 2012), which was replaced by Sentinel-1[8] in 2015 [1].

Other SAR sensors are already in orbit, such as the German TerraSAR-X and Tandem-X [9] and the Japanese ALOS-2 [10], while many others are currently being designed and tested for future launch (NovaSAR-S [11] and SAOCOM [12]). Furthermore, modern SARs operate in constellation and offer a wide spatial coverage. In future, it will be possible to synergically exploit the SAR images acquired from different sensors in order to drastically reduce the time elapsed between two consecutive observations of the same geographic area. In this way, a real time (RT) or near real-time (NRT) monitoring of the ocean and open sea will be possible.

Unfortunately, this successful upstream work has not been followed by an adequate exploitation of remote sensing data and relative downstream activities are still underdeveloped. The research presented in this paper is then timely and aims at helping to fill this gap by developing a novel ship detection algorithm based on the generalized-likelihood ratio test (GLRT) algorithm.

SAR is very capable in detecting targets over the sea surface due to the low backscattering of sea areas compared to that observed in presence of ships and vessels: when waters are flat and smooth, most of the incoming incidence wave is reflected in the specular direction providing a low backscattering coefficient for the observed marine areas. This is the main physical principle on which, so far, SAR ship-detection algorithms have been heavily based by means of a constant false alarm rate (CFAR) method. With CFAR, the sea clutter background is modeled according to a suitable distribution and a threshold is set to achieve an assigned PFA [13]. Several clutter distributions have recently been analyzed; however, CFAR algorithms are not able to detect targets with intensity values close to the sea clutter and the threshold computation represents 
a time-consuming procedure if a compound-Gaussian model is adopted [14].

In [15], a compound Gaussian CFAR filter is used in conjunction with a spatial distribution of the target, based on the kernel density estimator, in order to improve the detection of the bright targets. Results show a PFA and PD that are $0.2 \%$ lower and $1 \%$ higher than the standard CFAR probabilities, respectively.

Other studies are based on the sublook decomposition and analysis [16]-[19]. The original SAR image is divided into sublooks and then the coherence index between the sublooks is computed. Coherent targets show a higher coherence index, while the sea clutter pixels are usually uncorrelated. It has been shown that this technique improves the target-to-clutter ratio (TCR) up to $2 \mathrm{~dB}$ [16]. The main drawback of the sublook analysis is that the resolution is reduced depending on the number of sublooks employed (usually up to four). Conversely, on the positive side, no assumption is made about the sea clutter and how to model it. In [18], the resolution loss is partially overcome by computing the coherence index over partially overlapping sublooks. In [19], instead, Schneider et al. introduce an alternative approach to detect coherent scatterers over a clutter background based on the evaluation of the sublook entropy. The main difference, compared to the sublook correlation approach, is the greater number of sublooks employed for the evaluation of the sublook entropy, which provide more flexibility in the evaluation of the spectral correlation at the cost of a further resolution loss [19].

Finally, other approaches rely on fully polarimetric data showing an improved performance in target detectability [20]-[24]. There are some benefits in polarimetric detectors. For example, it has been shown in [23] that the TCR is higher for the cross-polarized channels than the copolarized ones for incidence angle lower than $50^{\circ}$. In [24], the four polarimetric channels are compared using a CFAR algorithm over a dataset acquired from an airborne platform operating at C-band. It has been demonstrated that the HV polarization performs the best among all the single polarization channels; in addition, the full polarimetric data (four channels) are superior to all other single and multipolarization configurations alone. Unfortunately, the availability of full polarimetric data is very limited and, consequently, the authors focus on the development of ship detectors with a single polarimetric channel.

In all the detectors already present in the literature, the target model (analytical and statistical) is ignored to avoid an increase in the complexity of the detectors. However, an optimal detector should take also the target into account, as suggested in [13]. In [25] and [26], Iervolino et al. introduced a novel scattering model for a canonical ship target. The double-reflection contribution has been characterized and a suitable distribution function for the signal backscattered from a canonical ship has been derived.

In this paper, starting from the outcomes derived in [25], Iervolino et al. build a novel ship-detection algorithm for single polarimetric SAR intensity images. The detection procedure relies on a GLRT based on the likelihood functions of both the sea clutter and the ship target. A model-based approach is employed in a SAR ship-detection chain for the first time in order to improve the overall performance.
This paper is organized as follows: In Section II, the derivation of the GLRT for the ship detection is explained along with the estimation methods to compute the clutter and target parameters; in Section III, the TCR is analytically evaluated at different bands (S-, C-, and X-) for a typical ship target; in Section IV, the GLRT and CFAR algorithms are compared through MonteCarlo simulations; in Section V, the ship detection algorithm is applied to real datasets acquired from different spaceborne and airborne sensors; finally, in Section VI, conclusions are drawn.

\section{GLRT DETECTOR}

In order to implement a GLRT, both the likelihood functions of the sea clutter and the target (the canonical ship in our case) must be defined. While the definition of a suitable distribution for the sea clutter is largely addressed in the literature [13]-[15], not much modeling of the ship backscatter has been undertaken in ship detection. In [27], for example, an extremely simple model for the ship target is presented to set a GLRT; the intensity pixels corresponding to the ship are assumed independent and Gaussian distributed with a zero mean, but no evidence is provided to support this assumption. Here, the distribution for the ship is derived from [25] where the double-reflection contribution (the main scattering mechanism occurring in the scenario ship/sea) was first analytically modeled and evaluated in closed forms (hence invertible) with the geometric optics (GO) theory within the Kirchhoff approximation in high-frequency regime. Successively, scene parameters appearing in the invertible formula were associated to a statistical distribution, thus transforming the originally deterministic model for the ship in a statistical one (see Section II-B). The latter is fundamental since the optimum statistical test relies on the target statistical distribution [28].

Before deriving the statistical test, it is possible to define the test hypotheses:

$$
\begin{aligned}
& H_{0} \text { : sea clutter } \\
& H_{1} \text { : canonical ship. }
\end{aligned}
$$

Differently from other detectors in the literature [29], [30], the background clutter is here related to the target distribution. Indeed, the hypothesis $H_{1}$ refers to the double-reflection contribution which occurs between sea surface and ship; hence, it is linked to the sea clutter through the roughness parameters of the GO model as highlighted in [31] and [32]. This scattering contribution has been already analyzed and its distribution was derived in [25].

The test which maximizes the PD for a given PFA (optimum test) is the likelihood ratio test (LRT), defined as follows [28]:

$$
\Lambda_{L}\left(\sigma_{x}\right)=\frac{p\left(\sigma_{x} / H_{1}\right)}{p\left(\sigma_{x} / H_{0}\right)}>\operatorname{Tr}(\mathrm{PFA}) \leftrightarrow \text { Detected }
$$

where $\Lambda_{L}(\cdot)$ is the likelihood ratio function, $\sigma_{x}$ is the radar cross section (RCS) of the pixel under test, $p\left(\sigma_{x} / H_{1}\right)$ and $p\left(\sigma_{x} / H_{0}\right)$ are the probability distribution functions (pdfs) of $\sigma_{x}$ given the presence of the target or of the clutter, respectively, and $\operatorname{Tr}(\mathrm{PFA})$ is the threshold according to the desired PFA. 
The LRT requires an explicit knowledge of the pdfs involved. However, in most of real cases, the parameters of the pdfs are unknown and need to be estimated. When these parameters are estimated through maximum-likelihood methods (MLE), the LRT becomes a GLRT defined as follows [28]:

$$
\Lambda_{G}\left(\sigma_{x}\right)=\frac{p\left(\sigma_{x} / H_{1}, \hat{\boldsymbol{\alpha}}_{t}\right)}{p\left(\sigma_{x} / H_{0}, \hat{\boldsymbol{\alpha}}_{c}\right)}>\operatorname{Tr}(\mathrm{PFA}) \leftrightarrow \text { Detected }
$$

where $\Lambda_{G}(\cdot)$ is the generalized likelihood ratio function, $\hat{\boldsymbol{\alpha}}_{t}$ and $\hat{\boldsymbol{\alpha}}_{c}$ are the MLE estimators relative to the target (hypothesis $H_{1}$ ), and the clutter (hypothesis $H_{0}$ ) distribution parameters, respectively. In the next sections, the methodology to compute the clutter and target distributions together with the corresponding parameters is shown along with a block diagram describing how to implement the GLRT algorithm.

\section{A. Clutter Estimation Parameters}

In order to statistically analyze the sea clutter, some assumptions have to be done.

1) The SAR return signal is made up of multiple scatterers in a resolution cell.

2) The amplitude and the phase of a single scatterer are statistically independent and identically distributed.

3) No scatterer is dominant.

4) The phase is uniformly distributed over all angles.

5) The number of scatterers in each resolution cell is large.

Within these hypotheses, the central limit theorem can be applied. As a consequence, the real and the imaginary parts of the return signal are independently and identically Gaussian distributed with zero mean and a variance denoted as $\sigma_{s}^{2} / 2$ [33]. The intensity of the SAR backscattered signal (proportional to the RCS) has a negative exponential distribution with scale parameter $\sigma_{s}^{2}$

$$
p\left(\sigma_{x} / H_{0}\right)=\frac{1}{\sigma_{s}^{2}} \exp \left(-\frac{\sigma_{x}}{\sigma_{s}^{2}}\right), \sigma_{x} \geq 0 .
$$

Under these hypotheses, the MLE of $\sigma_{s}^{2}\left(\hat{\sigma}_{s}^{2}\right)$ is the sample average

$$
\hat{\sigma}_{s}^{2}=\frac{1}{M} \sum_{i=1}^{M} \sigma_{x, i}
$$

where $M$ is the number of samples and $\sigma_{x, i}$ is the RCS of the $i$ th sample.

In addition, it is assumed that the sample average of the SAR intensity is equal to the RCS reflected by a rough surface within the GO approximation as already done by Iervolino et al. in [34]. In formula

$$
\hat{\sigma}_{s}^{2}=\sigma_{\mathrm{GO}}=\frac{\left|S_{p q}\right|_{c}^{2} a b}{64 \pi^{2} \cos ^{2} \vartheta \sigma_{\mathrm{dev}}^{2} / L^{2}} \exp \left(-\frac{t g^{2} \vartheta}{4 \sigma_{\mathrm{dev}}^{2} / L^{2}}\right) .
$$

In (6), $\sigma_{\mathrm{GO}}$ is the RCS relevant to the single scattering contribution of the sea clutter; $\left|S_{p q}\right|_{c}$ is the module of the generic element of the scattering matrix for the clutter, with $p$ and $q$ standing for horizontal $\mathrm{H}$ or vertical $\mathrm{V}$ polarization respectively; $\sigma_{\mathrm{dev}}$ and $L$ are the standard deviation and the correlation length, respectively, of the stochastic process representing the sea clutter; $\vartheta$ is the radar look angle and, finally, $a$ and $b$ are the dimensions of the rectangular portion of sea where the RCS is evaluated (generally set equal to the SAR spatial resolution). $\left|S_{p q}\right|_{c}$ depends on the dielectric constant of the sea $\left(\varepsilon_{\mathrm{SW}}\right), \vartheta$, the working wavelength $\lambda$ and the Fresnel coefficient according to the polarization of the propagating wave. Its explicit expression is provided in [31].

In case of heterogeneous clutter, the Gaussian distribution may not be the best model to approximate the heavy tail of the histogram and the $K$-distribution is usually preferred to model the sea clutter [35].

\section{B. Target Estimation Parameters}

The target distribution is computed starting from the evaluation of the RCS of the double-bounce contribution (thereflection mechanism arising between the ship hull and the sea clutter) within the GO-GO approximation [25], [31], [32]. In this scenario, the hull dimensions are much larger than the working radar wavelength $\lambda$ and the diffraction contributions can be neglected; the sea clutter, instead, is modeled via a Gaussian stochastic process as explained in the previous subsection. Single and multiple scattering contributions normally arise due to the particular configuration of the ship and the sea and can be properly modeled. However, with regard to the target, only the double scattering contribution is considered since it represents the dominant contribution from the ship [31].

For the sake of completeness, the final RCS formulations are here reported for the canonical target (GO-GO solution) [25]:

$$
\begin{aligned}
& \sigma_{\mathrm{GOGO}}= \\
& \frac{h\left|S_{p q}\right|_{t}^{2} l \tan \vartheta \cos \varphi\left(1+\tan ^{2} \vartheta \sin ^{2} \varphi\right) \exp \left[-\frac{\tan ^{2} \vartheta \sin ^{2} \varphi}{2 \sigma_{\mathrm{dev}}^{2}\left(2 / L^{2}\right)}\right]}{8 \pi^{2} \sigma_{\mathrm{dev}}^{2}\left(2 / L^{2}\right) \cos ^{2} \vartheta}
\end{aligned}
$$

where $\sigma_{\mathrm{GOGO}}$ is the RCS relevant to the double scattering contribution of the target for the GO-GO approximation; $\left|S_{p q}\right|_{t}$ is the module of the generic element of the scattering matrix for the ship target now, with $p$ and $q$ standing for horizontal $\mathrm{H}$ or vertical $\mathrm{V}$ polarization, respectively; $l$ is the length of the portion of the ship belonging to the resolution cell, assuming the ship length larger than the SAR spatial resolution; $\varphi$ is the angle between the sensor line of flight and the projection of the ship hull onto the water surface; $h$ is the height of the ship which contributes to form the dihedral surface between the sea and the ship hull (freeboard); $\left|S_{p q}\right|_{t}$ depends on the same parameters of $\left|S_{p q}\right|_{c}$ plus the dielectric constant of the hull $\left(\varepsilon_{H U L L}\right)$ and the orientation angle $\varphi$. Similarly as for $\left|S_{p q}\right|_{c}$, its explicit expression is provided in [31].

It is now possible to divide the input parameters of (7) into different categories.

1) Vector a: Parameters retrievable from the ancillary data of the SAR sensor. They are the radar look angle $(\vartheta)$, the spatial resolution $(\Delta x)$, and the radar wavelength $(\lambda)$.

2) Dielectric constant of the saline water $\left(\varepsilon_{S W}\right)$ computable from models presented in the literature for the particular radar parameters selected [36]. 


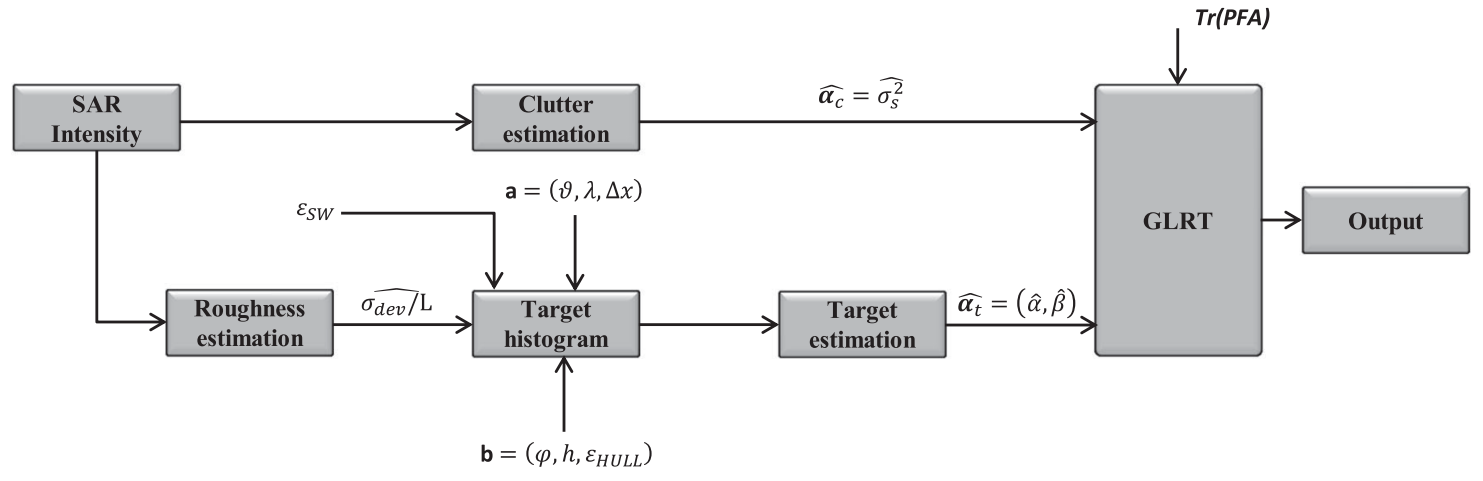

Fig. 1. Block diagram for a GLRT detector.

3) Roughness ratio $\left(\sigma_{\mathrm{dev}} / L\right)$ computable directly from the SAR image. It is evaluated by minimizing the absolute error between the RCS relevant to the single scattering of the sea surface and the RCS measured on the SAR image [34].

4) Vector b: Unknown parameters. They are the orientation angle $(\varphi)$, the freeboard height $(h)$, and the dielectric constant of the hull $\left(\varepsilon_{H U L L}\right)$.

In order to evaluate the RCS of the double-reflection contribution, suitable distribution functions are considered for each parameter of vector $\mathbf{b}$. In this way, the analytical model is turned in a statistical model, where the histogram of values relative to the double-reflection contribution can be computed. In order to evaluate and analyze this histogram, the same assumptions and distributions proposed in [25] are here considered. The histogram can be finally fitted with known distributions and it has been already demonstrated that the Gamma and the Weibull distributions best fit the histogram for the co- and crosspolarized channels, respectively [25].

In this paper, only datasets acquired with $\mathrm{HH}$ and VV polarization are considered; therefore, the Gamma distribution is used to model the target

$$
p\left(\sigma_{x} / H_{1}\right)=\frac{1}{\beta^{\alpha} \Gamma(\alpha)} \sigma_{x}^{\alpha-1} \exp \left(-\frac{\sigma_{x}}{\beta}\right), \sigma_{x} \geq 0
$$

where $\alpha$ and $\beta$ are the shape and the scale parameter, respectively.

Numerical methods are needed to obtain the MLE for the Gamma family of random variables. In particular, the MATLAB MLE function is applied here; it relies on the Newton-Raphson method which aims at maximizing the score function (log likelihood).

\section{GLRT Block Diagram}

The block diagram to implement the GLRT is shown in Fig. 1. The diagram is made up of two branches: one to estimate the clutter parameters $\boldsymbol{\alpha}_{c}$ and the other one to estimate the target parameters $\boldsymbol{\alpha}_{t}$. First, a clutter region of interest (ROI) is isolated and analyzed. In the clutter estimation block, assuming an exponential intensity distribution, the clutter parameter is estimated by computing the sample average of the ROI. The roughness parameters $\left(\sigma_{\mathrm{dev}} / L\right)$ are estimated in the roughness estimation block by minimizing the absolute error between the theoretical

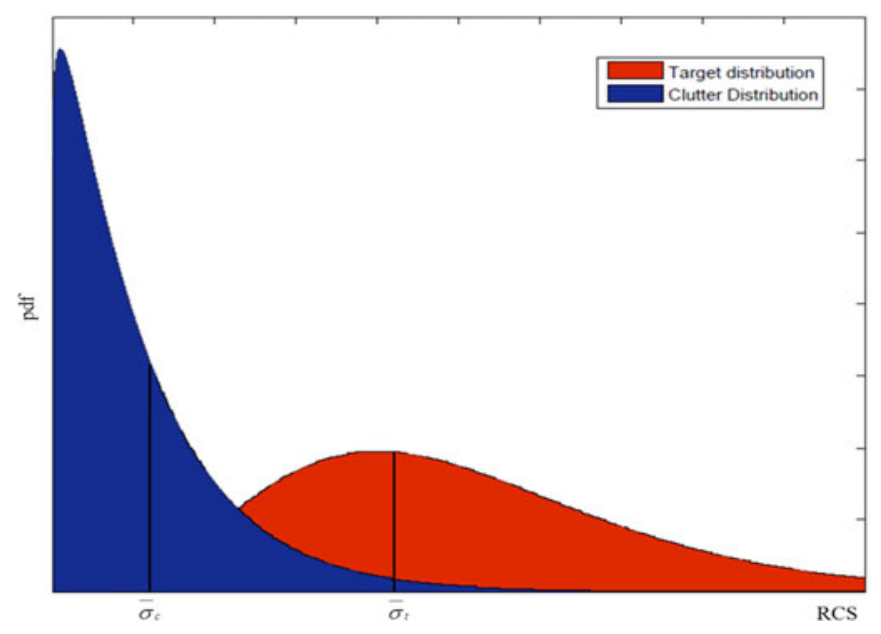

Fig. 2. RCS distribution relative to the sea clutter and the ship target in a canonical scenario

RCS of the clutter within GO approximation and the RCS directly measured on the SAR intensity image. These parameters represent an input along with the vectors $\mathbf{a}=(\vartheta, \lambda, \Delta x)$ and $\mathbf{b}=\left(\varphi, h, \varepsilon_{H U L L}\right)$ and the dielectric constant of the saline water $\left(\varepsilon_{\mathrm{SW}}\right)$ to the target histogram block. The latter comprises the computation of the histogram relevant to the double-reflection contribution arising between the ship hull and the sea clutter. The target parameters $(\alpha, \beta)$ of the Gamma distribution are estimated in the target estimation block by using numerical methods as mentioned in the previous section.

The GLRT is then performed according to a desired PFA, which is an input to the GLRT block. Finally, the pixels with a $\Lambda_{G}(\cdot)$ greater than the fixed threshold are detected.

Similarly to the CFAR, the estimation procedures can be done by using a moving window and computing the clutter and the target parameters along with the threshold at each iteration (adaptive threshold algorithm). Vice versa, the target and clutter parameters can be estimated for a single representative ROI leading to a fix threshold (global threshold algorithm).

The GLRT is not the optimum statistical test but, if the pdfs of both clutter and target are well defined, it can improve the TCR as shown in Fig. 2. Indeed, considering the mean clutter RCS value $\left(\bar{\sigma}_{c}\right)$ it results $\Lambda_{G} \ll 1$, while $\Lambda_{G} \gg 1$ when considering the mean target RCS value $\left(\bar{\sigma}_{t}\right)$. Consequently, the output of the GLRT is an image where the clutter is attenuated and the targets 
TABLE I

PARAmeters VAlues NeEded to COMPUte the ANAlytical TCR

\begin{tabular}{lc}
\hline \hline Parameters & Value \\
\hline Radar look angle $\vartheta[\mathrm{deg}]$ & $40^{\circ}$ \\
Radar working frequency $f[\mathrm{GHz}]$ & {$[3.2 ; 5.4 ; 9.6]$} \\
Roughness parameters $\sigma_{\mathrm{dev}} / L$ & 0.2 \\
Length of the portion of the ship $l[\mathrm{~m}]$ & 10.0 \\
Sea portion $a[\mathrm{~m}]$ & 10.0 \\
Sea portion $b[\mathrm{~m}]$ & 10.0 \\
Dielectric constant of the sea $\varepsilon_{\mathrm{SW}}$ & Reference $[36]$ \\
Salinity $S a \% 0$ & {$[30] ;[35] ;[40]$} \\
Dielectric constant of the ship hull $\varepsilon_{H} U L L$ & Equation $(10), p=0.7 q=0.3$ \\
Orientation angle $\varphi[\mathrm{deg}]$ & $5^{\circ}$ \\
Freeboard height $h[\mathrm{~m}]$ & 3.0 \\
\hline \hline
\end{tabular}

are enhanced. Therefore, it results that after the thresholding step, the performance of the GLRT is better than the standard CFAR algorithm.

However, the GLRT needs to estimate the target parameters vector $\left(\boldsymbol{\alpha}_{t}\right)$ and not only the clutter parameters vector $\left(\boldsymbol{\alpha}_{c}\right)$ as CFAR algorithms do. As a consequence, the GLRT presents a computational load higher than CFAR algorithms as shown by the simulations in Section IV.

In the next section, the analytical TCR is evaluated for a typical target within the GO solution at different bands (S-, C-, and $\mathrm{X}$ ).

\section{TCR FOR A CANONICAL SHIP TARGET}

In this section, the authors compare the TCR of a typical ship target at different bands (S-, C-, and X-) with the purpose of identifying the most suitable band for ship detection in SAR imagery; in fact, the higher the TCR, the better the detector's performance. As a result, a method to improve the ship-detection performance is to maximize the TCR [37].

The TCR can be expressed as a ratio of the target RCS $\left(\sigma_{\mathrm{GOGO}}=\sigma_{\text {target }}\right)$ to the sea clutter RCS $\left(\sigma_{G O}=\sigma_{\text {clutter }}\right)$, [25], [34]:

$$
\mathrm{TCR}=\frac{\sigma_{\mathrm{GOGO}}}{\sigma_{\mathrm{GO}}}=\frac{\sigma_{\text {target }}}{\sigma_{\text {clutter }}} .
$$

Before computing the TCR, some assumptions about the dielectric parameters are required. In this study, $\varepsilon_{\mathrm{SW}}$ is computed according to the saline-water double-Debye dielectric model in [36]: it is a function of the water salinity $\left(S_{a}\right)$, water temperature $(T)$, and the sensor working frequency $(f) . \varepsilon_{H U L L}$ is evaluated by performing a weighted average of several dielectric constants which compose the typical hull of a ship [25], [38]. Here, it is assumed that the canonical target is made of steel $(70 \%)$, glass $(10 \%)$, aluminum (10\%), and fused silica (10\%), therefore $\varepsilon_{H U L L}$ can be computed according to the following:

$$
\varepsilon_{H U L L}=p \varepsilon_{s t}+\frac{q}{3}\left(\varepsilon_{a}+\varepsilon_{g}+\varepsilon_{s i}\right)
$$

where $p=0.7, q=0.3, \varepsilon_{s t}, \varepsilon_{a}, \varepsilon_{g}$, and $\varepsilon_{s i}$ are the complex relative dielectric constants of steel, aluminum, glass, and fused silica, respectively. Their values vary in frequency and are reported in [25] for all the bands considered in this paper.

In Table I, all the parameters used to compute the TCR are reported. Finally $\left|S_{H H}\right|_{t},\left|S_{H H}\right|_{c}$ and the relative TCR (in dB) are
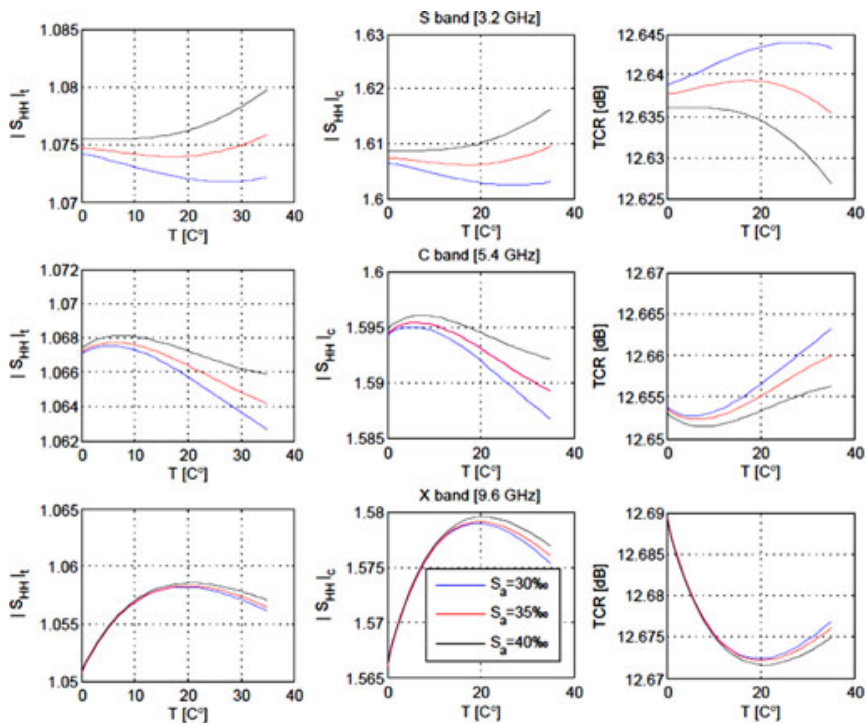

Fig. 3. $\left|S_{H H}\right|_{t},\left|S_{H H}\right|_{c}$, and TCR at $\mathrm{S}$ band (top row), $\mathrm{C}$ band (middle row), and $\mathrm{X}$ band (bottom row) against the sea temperature for different value of the water salinity.

plotted in Fig. 3 at different bands $(\mathrm{S}=3.2 \mathrm{GHz}, \mathrm{C}=5.4 \mathrm{GHz}$, and $\mathrm{X}=9.6 \mathrm{GHz}$ ) for $\mathrm{HH}$ polarization by letting the water salinity and temperature vary. The average values of the water temperature and salinity can be retrieved from the World Ocean Atlas 2009 Figures (WOA09F) available at [39]. In this study, no assumption is made about the particular area where the SAR images could be acquired and, therefore, the ranges of water temperature and salinity are set as wide as possible after analyzing the WOA09F atlas $\left(T=\left[0{ }^{\circ} \mathrm{C} ; 35^{\circ} \mathrm{C}\right]\right.$ and $S_{a}=$ $30 \% ; 40 \%$ ) . In general, it results that the backscattering coefficients of both target and clutter slightly change with the variation of water temperature and salinity providing that the TCR is about $12.6 \mathrm{~dB}$ for all the working frequency analyzed. As a consequence, we do not expect a significant variation in the performance of the ship detector with a frequency variation, as the next section demonstrates.

\section{Monte-Carlo Simulations}

In this section, the new GLRT technique is compared to the standard CFAR algorithm through simulations. At this aim, several SAR images are simulated trough a Monte-Carlo approach [33] as shown in the flowchart in Fig. 4.

First, $200 \times 200$ sea clutter pixels are simulated with a MonteCarlo method in the sea scattering block assuming an exponential distribution for the clutter intensity, whose mean value is set equal to the single scattering contribution from a rough sea surface within the GO approximation of (6) $\left(\sim \operatorname{Exp}\left(\sigma_{\mathrm{GO}}\right)\right)$. The sea scattering block takes radar parameters $(f$ and $\vartheta)$, roughness parameters $\left(\sigma_{\mathrm{dev}}\right.$ and $\left.L\right)$, and the dielectric constant of the saline water $\left(\varepsilon_{\mathrm{SW}}\right)$ to produce the mean value of the exponential distribution. Second, all the pixels with normalized RCS (NRCS) values lower than a typical noise equivalent signal zero (NESZ) of the SAR sensor are set equal to the NESZ producing the $i_{c}(x, r)$ image, where $x$ and $r$ represent the azimuth and range distance, respectively. In all simulations, it has been 


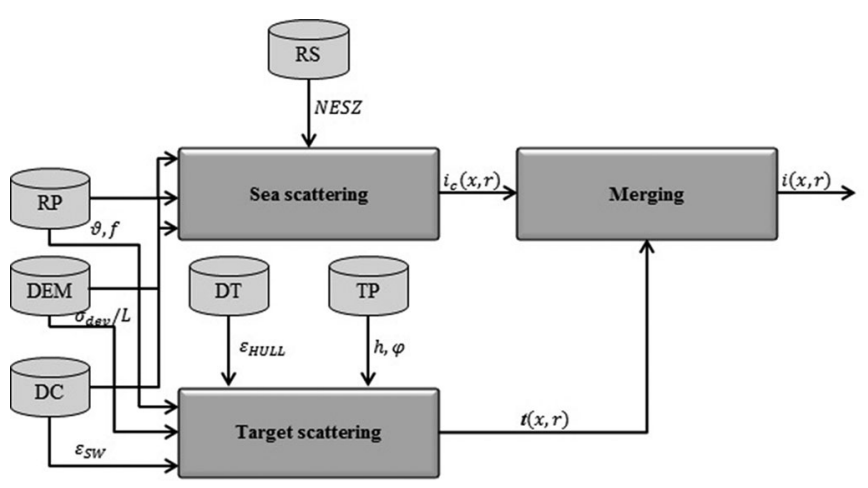

Fig. 4. Block diagram for the SAR images simulation. The cylindrical blocks represent the memories where the input parameters are stored: Radar parameters (RP), digital elevation model (DEM), dielectric constant of clutter (DC), dielectric constant of target (DT), target parameters (TP), and radar sensitivity (RS). The rectangular blocks are the processing operations.

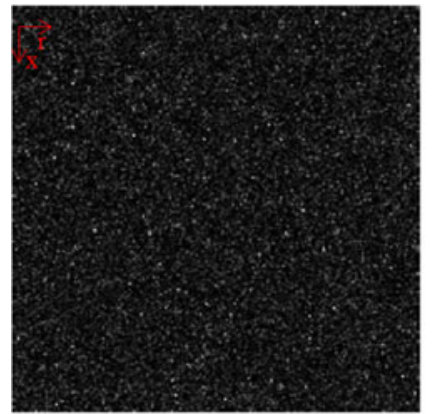

(a)

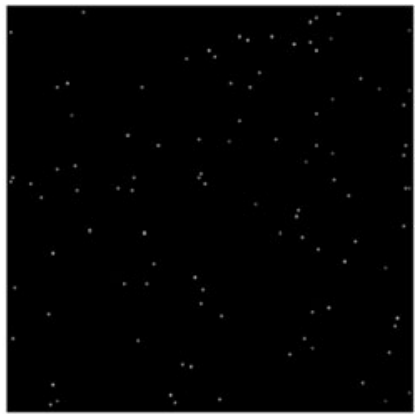

(b)
Fig. 5. Simulated SAR Intensity image in slant range $(r)$ /azimuth $(x)$ plane (a). Relative detection mask where the dark pixels represent the sea clutter and the bright pixels the ship targets (b).

assumed a typical NESZ of $-25 \mathrm{~dB}$, which represents the mean value for the TerraSAR-X sensor [40]. However, the sensitivity of most SAR sensors can be bound between $-20 \mathrm{~dB}$ (worst case) and $-30 \mathrm{~dB}$ (best case) [36]; so we can assume a mean value of $-25 \mathrm{~dB}$ for the different bands without the loss of generality. Third, in the target scattering block, 100 target RCS values are simulated according to the canonical model presented in [25]. This block takes as input the same parameters of the sea scattering block plus the dielectric constant of the hull $\left(\varepsilon_{H U L L}\right)$ and the target parameters ( $h$ and $\varphi$ ) producing the vector $t(x, r)$ as output. In this block, targets are always simulated as single point scatterers. In the last step, the simulated targets are placed in random positions in the $i_{c}(x, r)$ image through the merging block. In this way, the final $i(x, r)$ SAR image, which can feed the detector block shown in Fig. 1, is obtained. Finally, a SAR simulated image is reported in Fig. 5(a), while the relative detection mask is shown in Fig. 5(b) where the dark pixels (39 900 in total) represent the sea clutter and the bright ones the ship targets (100 in total).

First of all, the GLRT and the CFAR algorithms are compared at S-, C-, and X-bands in a typical scenario: the ROC curves are derived by computing the PD and PFA for several threshold values and, then, the computational load is evaluated for both approaches. The radar and roughness parameters set to simulate the SAR images are the same ones used to analytically evaluate
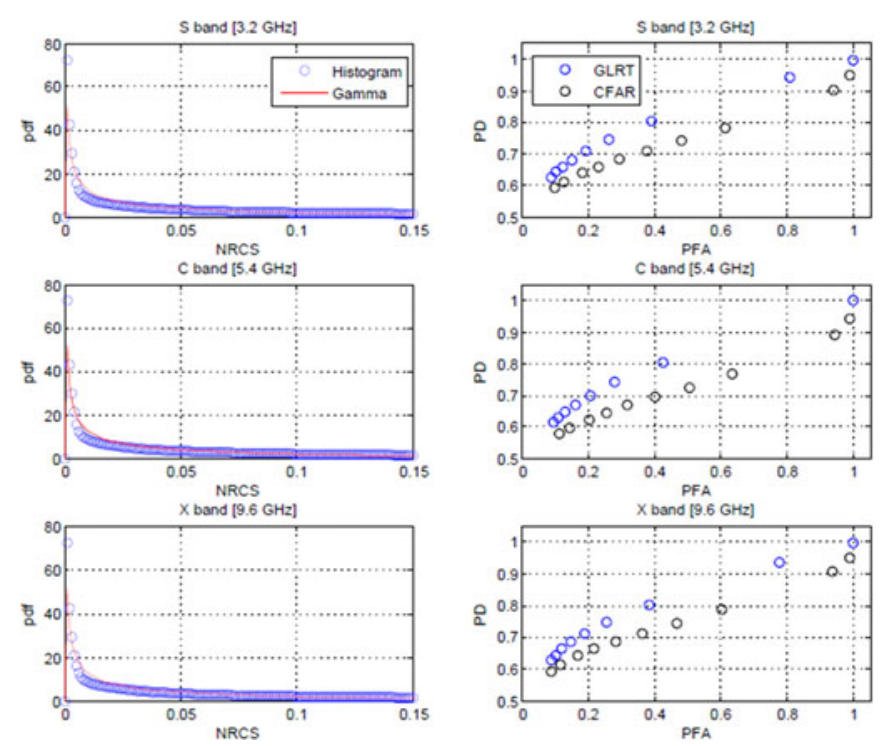

Fig. 6. Histogram of the NRCS relevant to the double-reflection contribution for $\mathrm{HH}$ polarization and the Gamma distribution on the left side. ROC curves comparison between the GLRT and CFAR detectors on the right side at $\mathrm{S}$ band (top row), $\mathrm{C}$ band (middle row), and $\mathrm{X}$ band (bottom row).

TABLE II

Mean Value and Standard DeViation of the NRCS Along With the $P$ Value $(\%)$ RELATIVE TO THE $\chi^{2}$ GOF TEST AT HH POLARIZATION FOR EACH BAND

\begin{tabular}{lccc}
\hline \hline Parameter & \multicolumn{3}{c}{ Band } \\
\cline { 2 - 4 } & $\mathrm{S}$ & $\mathrm{C}$ & $\mathrm{X}$ \\
\hline Mean NRCS & 0.088 & 0.086 & 0.0854 \\
std NRCS & 0.110 & 0.108 & 0.107 \\
$p$ value (\%) & 53.89 & 77.34 & 80.17 \\
\hline \hline
\end{tabular}

the TCR in the previous section (see Table I). Furthermore, the values $T=20^{\circ}$ and $S_{a}=35 \%$ o have been assumed for the characterization of the sea clutter.

In Fig. 6 (left panel), the pdfs relative to the histogram and the fitting with a Gamma distribution are shown for $\mathrm{HH}$ polarization at S-, C-, and X-bands. The histograms are relative to the NRCS defined as

$$
\sigma^{0}=\frac{\sigma}{\Delta x \Delta g}
$$

where $\sigma^{0}$ is the NRCS, $\Delta x$ and $\Delta g$ are the azimuth and ground range resolution, respectively, which are set equal to $a$ and $b$ from Table I $(10 \mathrm{~m})$. The Gamma distribution passes the $\chi^{2}$ goodness of fit test [41] for all the bands and so it can be used to model the backscattering return of the double-reflection contribution of the canonical target. The relative $p$ values along with the mean value and the standard deviation of the histogram are reported in Table II. As expected, the results exhibit a negligible dependence with respect to the frequency variation and, consequently, the detectors performance will be also similar.

In Fig. 6 (right panel), the ROC curves of GLRT and CFAR methods are retrieved after 100 tests for each threshold. In general, it results that the GLRT-based technique performs better 
TABLE III

CFAR AND GLRT COMPUTATIONAL TIMES FOR ANALYZING 400000 PIXELS

\begin{tabular}{lcc}
\hline \hline Operation & CFAR [s] & GLRT [s] \\
\hline Clutter parameters estimation & 0.296 & 0.296 \\
Target parameters estimation & n.a. & 1.385 \\
GLRT evaluation & n.a. & 0.193 \\
Thresholding & 0.361 & 0.361 \\
TOT & 0.657 & 2.235 \\
\hline \hline
\end{tabular}

(higher PD for any given PFA) than for the standard CFAR algorithm for each band analyzed. For example, fixing PFA = $10^{-1}$ and performing a quadratic interpolation, the $\mathrm{PD}$, for the GLRT and CFAR, respectively, results: $\mathrm{PD}=0.64$ and $\mathrm{PD}=$ 0.60 at $\mathrm{S}$-band; $\mathrm{PD}=0.63$ and $\mathrm{PD}=0.58$ at $\mathrm{C}$-band; $\mathrm{PD}=0.65$ and $\mathrm{PD}=0.61$ at $\mathrm{X}$-band. The best performance is obtained at X-band for both CFAR and GLRT; however, it is clear that all the bands are almost equally good and there is not really a more convenient range of frequencies in which the detectors performance significantly improves.

In Table III, the computational times to derive a single dot (coming from the processing of $4 \times 10^{5}$ pixels), relative to the simulations shown in Fig. 6 for both the CFAR and GLRT, are reported. Using an Intel Pentium i5-2400 processor at 3.10 $\mathrm{GHz}$, it results that the computational time of the CFAR is three times smaller than the GLRT one because it does not need to estimate the target parameters [42]. However, it is important to underline that clutter and target parameters need to be estimated only once in a global thresholvd algorithm [13], while the thresholding phase (the most time demanding step) is directly proportional to the number of pixels to process. As a consequence, the difference in the computational load between the CFAR and GLRT decreases as the pixels of the image increase.

It must be pointed out that the comparison between GLRT and CFAR in this simulating section is biased by the fact that the targets are generated according to the distribution included in the GLRT model. A different choice may be done if the ground truth relative to thousands ships is available and the target can be generated according to real RCS values. However this level of ground truth was not available at this stage and the targets RCS were simulated according to the electromagnetic model introduced in [25].

Other simulations have been performed by letting the radar look angle $(\vartheta)$ and the roughness parameters vary $\left(\sigma_{\mathrm{dev}} / L\right)$ in order to get a complete picture of the GLRT detector performance. The variation of the dielectric constant of the hull $\left(\varepsilon_{H U L L}\right)$ is not considered in the ROC curves because it has been demonstrated in [25] that the RCS relative to the canonical ship is robust to the variation of permittivity and conductivity of the materials composing the ship hull. Only the X-band at $\mathrm{HH}$ polarization and the GLRT algorithm are considered in the following simulations. Results are shown in Fig. 7.

Looking at the ROC curves on the left panel of Fig. 7, where the radar look angle is fixed $\left(40^{\circ}\right)$ and the roughness ratio varies, it is shown that the performance gets worse when $\sigma_{\mathrm{dev}} / L$ increases because the radar backscattering from the sea clutter increases due to a rougher surface and, consequently, the TCR is reduced.
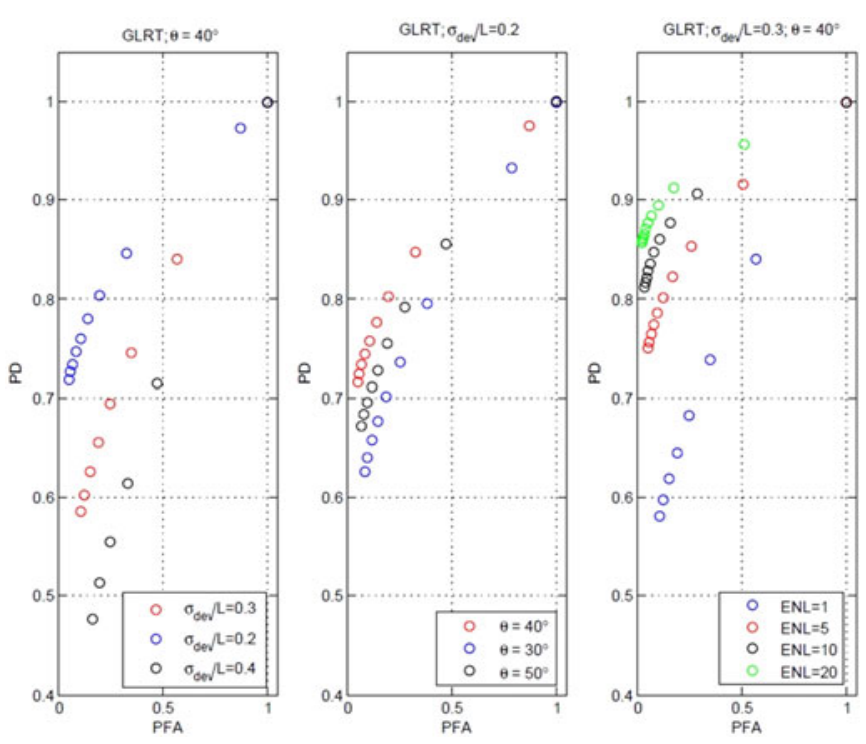

Fig. 7. ROC curves relative to the GLRT at $\mathrm{X}$ band and $\mathrm{HH}$ polarization for different value of the roughness ratio $\left(\sigma_{\mathrm{dev}} / L\right)$ on the left panel, different radar look angles $(\vartheta)$ in the middle panel and different equivalent number of looks (ENL) on the right panel.

Looking at the ROC curves in the middle panel of Fig. 7, where $\sigma_{\mathrm{dev}} / L$ is fixed (0.2) and the radar look angle varies, it is highlighted that the performance gets better with an increase of the radar look angle because most of the incidence radiation of the sea clutter is reflected in the specular direction. However, for very high look angles $\left(\vartheta \geq 50^{\circ}\right)$ also, the RCS relevant to the target double-reflection contribution diminishes and the TCR does not further increase. As a consequence, the performance of the detectors at $\vartheta=50^{\circ}$ is worse than that experienced at $\vartheta=40^{\circ}[42]$.

Finally, looking at the right panel of Fig. 7, the ROC curves are derived by fixing $\sigma_{\mathrm{dev}} / L$ and $\vartheta$ and multilooking the SAR simulated data. In the case of fully developed speckle, each clutter contribution is independent from others and the clutter can be seen as a collection of random variables that are independent and identically distributed. Within these hypotheses, the resulting clutter (after the multilooking operation) is Gamma distributed $\sim \Gamma\left(\mathrm{ENL} ; \sigma_{\mathrm{GO}} / \mathrm{ENL}\right)$ [33] and the speckle is reduced by a factor $1 / \sqrt{\mathrm{ENL}}$ [25], [32], where ENL represents the equivalent number of looks. It can be observed that the performance improves sharply with the increase of ENL because the multilooking reduces the speckle of the sea clutter, while the behavior of the target response is unaltered because the canonical ship is a coherent scatterer. In other words, when performing a multilooking operation, the standard deviation of the sea clutter distribution is multiplied by $1 / \sqrt{\mathrm{ENL}}$ while the mean value is unchanged; consequently, since the clutter distribution becomes more concentrated around its mean value, it is less likely that some clutter pixels have RCS greater than the targets RCS and the detector has a much higher PD at a fixed PFA. For example, considering PFA $=10^{-1}$, PD increases from 0.58 for $\mathrm{ENL}=1$ to 0.89 for $\mathrm{ENL}=20$. Conversely, with the increase of the ENL, the spatial resolution is reduced by a factor 1/ENL; consequently, ENL has to be chosen according to the minimum size of the targets that have to be detected and accord- 
TABLE IV

SAR ACQUISITIONS PARAMETERS

\begin{tabular}{lcccc}
\hline \hline Parameter & TerraSAR a & TerraSAR b & Sentinel-1 & Airbus dem. \\
\hline Acquisition Date & $09 / 11 / 12$ & $07 / 06 / 2016$ & $01 / 12 / 14$ & $28 / 07 / 10$ \\
Acquisition Time & $17: 52$ & $06: 26$ & $17: 48$ & $15: 38$ \\
Data Type & SLC & MGD & GRD & SLC \\
Number of azimuth pixels & 5336 & 12000 & 3500 & 1298 \\
Number of range pixels & 2869 & 5500 & 5500 & 1165 \\
Azimuth Resolution [m] & 3.30 & 16.5 & 23 & 0.84 \\
Range Resolution [m] & 1.77 & 16.5 & 23 & 0.84 \\
Azimuth pixel spacing [m] & 1.90 & 16.5 & 10 & 0.35 \\
Range pixel spacing [m] & 1.36 & 16.5 & 10 & 0.35 \\
Radar look angle [deg] & $41^{\circ}$ & $32^{\circ}$ & $35^{\circ}$ & $7^{\circ}-41^{\circ}$ \\
Working frequency [GHz] & 9.65 & 9.65 & 5.41 & {$[3.20 ; 9.60]$} \\
Polarization & $\mathrm{HH}$ & $\mathrm{VV}$ & $\mathrm{HH}$ & $\mathrm{HH}$ \\
Number of looks & $1 \times 1$ & $16 \times 2$ & $6 \times 6$ & $1 \times 1$ \\
\hline \hline
\end{tabular}

ing to the original spatial resolution. In real scenarios, the ship targets are not single point scatterers and the target intensity is spread over the size of the averaging windows employed for the multilooking operation. If the ship length is lower than the averaging window length, the TCR is reduced after multilooking, thus negatively affecting the detector performance. As a general advice, the higher the original spatial resolution is, the higher ENL can be chosen.

Finally, it is important to underline that the simplified assumptions made to model the canonical target lead to an underestimation (about $1.5 \mathrm{~dB}$ at X-band for $\mathrm{HH}$ polarization) of the real RCS as demonstrated in [25]. However, this underestimation can be regarded as a minor issue, meaning that targets in real scenario are more easily detectable and the detector performance is consequently higher than the one computed in this section through statistical simulations.

In the next section, the GLRT algorithm is finally tested over real SAR images acquired from different sensors.

\section{OUTCOMES ON REAL DATASETS}

The novel GLRT algorithm is tested and compared against the CFAR on four SAR meaningful datasets: two acquired from TerraSAR-X at X-band over the Solent Channel in U.K., one acquired from Sentinel-1 at C-band over the Portsmouth harbor and the last one acquired from the Airbus airborne SAR demonstrator simultaneously at S- and X-bands over the Angle Bay in the Pembrokeshire in U.K. The acquisition parameters for each dataset are reported in Table IV.

First of all, SAR images are processed in order to distinguish between land and sea pixels. In particular, land masking performed by employing shuttle radar topography mission 3 -arc-second data (approximately 90-m resolution) and by using the Sentinel-1 tool [44] developed by the European Space Agency to deal with SAR images.

In the following, the detectors outcomes are compared, when possible, with AIS data retrieved from [43]. Unfortunately, as already underlined in [25] and [26], the available ground truth looks incomplete because more ship signatures are visible in the SAR images than those recorded with the AIS. As a consequence, it is not possible to retrieve the PFA and the PD from the real datasets. However, the GLRT and CFAR are compared in terms of computational time and TCR. The TCR is evaluated as follows:

$$
\mathrm{TCR}=\frac{E\left[\left(\hat{\sigma}_{j}^{0}\right)_{T}\right]}{E\left[\left(\hat{\sigma}_{k}^{0}\right)_{C}\right]}=\frac{\frac{1}{N_{T}} \sum_{j=1}^{N_{T}}\left[\frac{1}{N_{j}} \sum_{i=1}^{N_{j}}\left(\hat{\sigma}_{i j}^{0}\right)_{T}\right]}{\frac{1}{N r} \sum_{k=1}^{N r}\left[\frac{1}{N a} \sum_{m=1}^{N a}\left(\hat{\sigma}_{m k}^{0}\right)_{C}\right]}
$$

where $E[\cdot]$ represents the mean operator, $\left(\hat{\sigma}_{j}^{0}\right)_{T}$ is the NRCS of the $j$ th ship, and $\left(\hat{\sigma}_{k}^{0}\right)_{C}$ is the NRCS of the $k$ th clutter range line. At numerator, $\left(\hat{\sigma}_{i j}^{0}\right)_{T}$ is the intensity of the $i$ th pixel associated to the double-reflection contribution of the $j$ th ship, $N_{j}$ is the number of resolution cells in the double-reflection line relative to the $j$ th ship $\left(N_{j}=l_{T j} / \Delta x\right.$ where $l_{T j}$ is the length of the $j$ th ship), and $N_{T}$ is the number of targets with AIS data available. At denominator, $\left(\hat{\sigma}_{m k}^{0}\right)_{C}$ is the intensity of the clutter pixels whose coordinates are $(m, k)$, while $\mathrm{Nr}$ and $\mathrm{Na}$ are the numbers of clutter pixels analyzed in the range and azimuth coordinates, respectively.

The TCR is evaluated before and after performing the GLRT [see (3)] and, consequently, the TCR gain can be computed.

\section{A. TerraSAR-X Datasets}

The first dataset used to test the GLRT algorithm is a single look complex (SLC) Stripmap image acquired from the TerraSAR-X sensor (X-band) over the Solent area in UK on 9th November, 2012. In Fig. 8(a), the intensity of the SAR image is shown, while masking results are displayed in Fig. 8(b) where the white pixels represent the land (masked out from the following processing steps) and the black ones correspond to the sea areas.

First of all, the image is calibrated according to the process described in [40] and by retrieving the information about the TerraSAR-X NESZ and the absolute calibration constant from the ancillary SAR data.

In Fig. 8(c), the GLRT image is shown after computing the $\Lambda_{G}(\cdot)$ function and assuming the clutter Gaussian distributed with the relative intensity exponentially distributed and the ship target Gamma distributed according to the electromagnetic model presented in [25] and summarized in Section II. Fig. 9 shows the details about the goodness of fit concerning the sea clutter, where a ROI of $400 \times 400$ pixels has been isolated and highlighted with the yellow rectangle in Fig. 8. It has been figured out that the exponential distribution passes the $\chi 2$ test and can be used to model the sea clutter. In the same ROI, the roughness ratio $\sigma_{\mathrm{dev}} / L$ has been computed by minimizing the absolute error between the RCS relevant to the single scattering of the sea surface and the RCS directly measured on the SAR image. The methodology is explained in [34], where the results (see [34, Fig. 2]) are also shown for the considered dataset. The ratio $\sigma_{\operatorname{dev}} / L$ is then used as an input along with all the other parameters of (7) to compute the histogram of the RCS relevant to the double-reflection contribution. This histogram is finally fitted with known distributions and it has been demonstrated that the Gamma distribution best fits this histogram (see [25, Fig. 7]). 


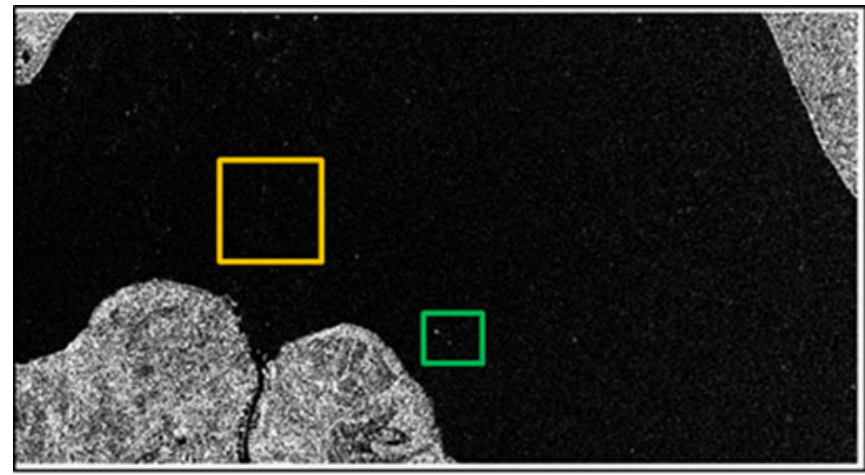

(a)

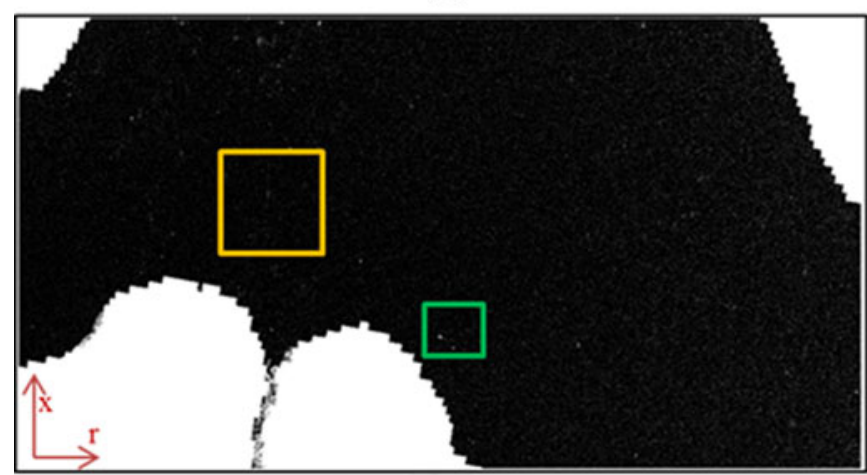

(b)

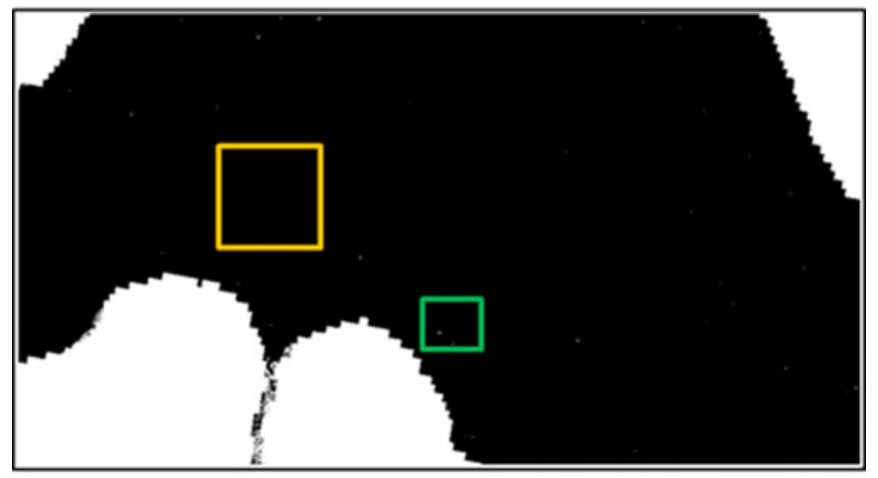

(c)

Fig. 8. SAR intensity image from the TerraSAR-X dataset acquired in $\mathrm{HH}$ polarization and $\mathrm{X}$ band on $09 / 11 / 12$ in slant range $(r)$ /azimuth $(x)$ plane (a). Land masking outcomes (b). GLRT SAR image (c). The green rectangle is the ROI isolated to analyze the TCR, while the yellow rectangle is the ROI isolated to estimate the clutter parameter and to compute the roughness ratio $\sigma_{\mathrm{dev}} / L$.

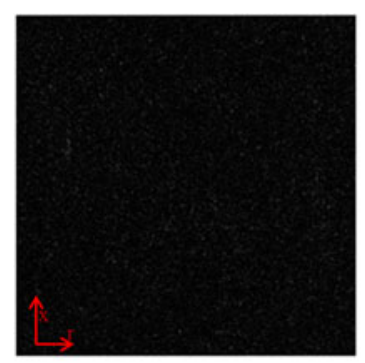

(a)

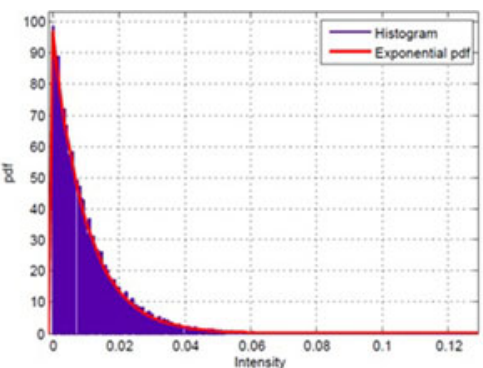

(b)
Fig. 9. (a) Yellow ROI Intensity image from TerraSAR-X sensor (a). Fitting of the exponential distribution to the clutter intensity histogram (b).
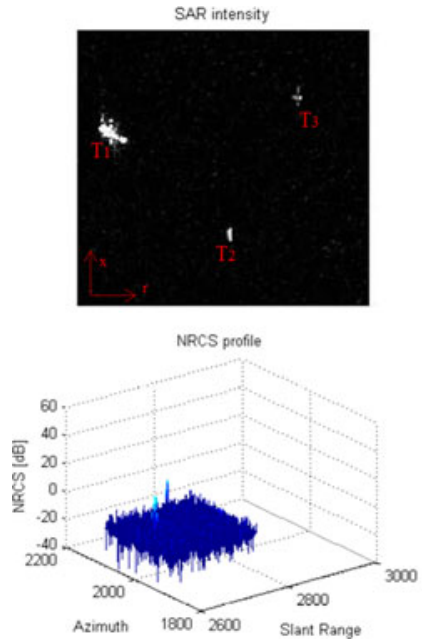

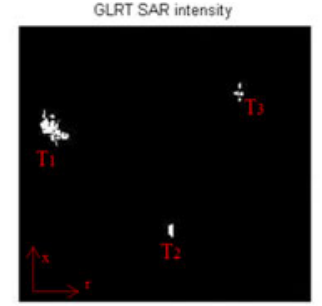

GLRT NRCS profile

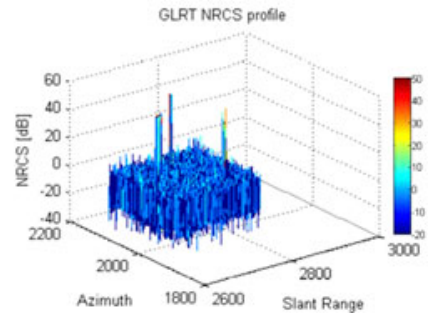

Fig. 10. Green ROI intensity image from TerraSAR-X sensor before and after applying the generalized-likelihood function band in slant range (r)/azimuth $(x)$ plane at the top. $T_{1}, T_{2}$, and $T_{3}$ are the targets signatures. NRCS profile relative to the ROI, before and after applying the generalized-likelihood function at the bottom.

From a visual inspection of the SAR image in Fig. 8(c), it results that the clutter is attenuated as it appears darker, while the targets are enhanced. In order to quantify the improvement in the TCR, a ROI of $200 \times 190$ pixels, corresponding to $380 \mathrm{~m} \times 258 \mathrm{~m}$ in azimuth and slant range, respectively, is isolated. The ROI is highlighted with a green rectangle in Fig. 8 and includes the signature of two out of seven available AIS signals.

A SAR intensity image relative to the selected ROI is shown at the top panel of Fig. 10, before and after applying the generalized-likelihood function. Conversely, the relative NRCS profile evaluated in decibel is shown at the bottom panel of Fig. 10. At this point, it is possible to evaluate the TCR relative to the original image and the enhanced image by applying (10). In Fig. 10, $T_{1}$ and $T_{2}$ are the signatures of the targets with the AIS data available, while $T_{3}$ is the signature of a much smaller craft without AIS signal. It has been computed that the average TCR value varies from 16.7 to $43.3 \mathrm{~dB}$ before and after applying the $\Lambda_{G}(\cdot)$ function with an increment of $26.6 \mathrm{~dB}$. It is clear from this analysis that the GLRT algorithm is able to detect ships with an RCS very close to the sea clutter RCS; hence, it is expected to retrieve more targets than a standard CFAR when the same PFA is fixed for both algorithms.

A PFA of $10^{-7}$ with a global threshold is employed at the detector stage for both the GLRT and CA-CFAR cell averaging CFAR (CA-CFAR) [35] algorithms for all the datasets used in this paper.

The performance of both detectors is finally shown in Table V. Both the GLRT and CFAR have correctly detected the AIS signals available (seven targets at all); however, the GLRT is able to detect more targets (54 against 33 retrieved by CFAR) since it presents a much better TCR ( $49.9 \mathrm{~dB}$ against $21.2 \mathrm{~dB})$ as already underlined in the analysis of Fig. 10. The TCR is evaluated by computing (12) for the seven AIS signals. The extra targets detected with the GLRT algorithms have been checked through a visual inspection of the relative signatures on the SAR image. 
TABLE V

CFAR AND GLRT PERFORMANCE COMPUTED ON TERRASAR A DATASET

\begin{tabular}{lcc}
\hline \hline & CFAR & GLRT \\
\hline TCR [dB] & 21.2 & 49.9 \\
AIS detected & 7 & 7 \\
Total targets detected & 33 & 54 \\
Computational time [s] & 31.5 & 48.3 \\
\hline \hline
\end{tabular}

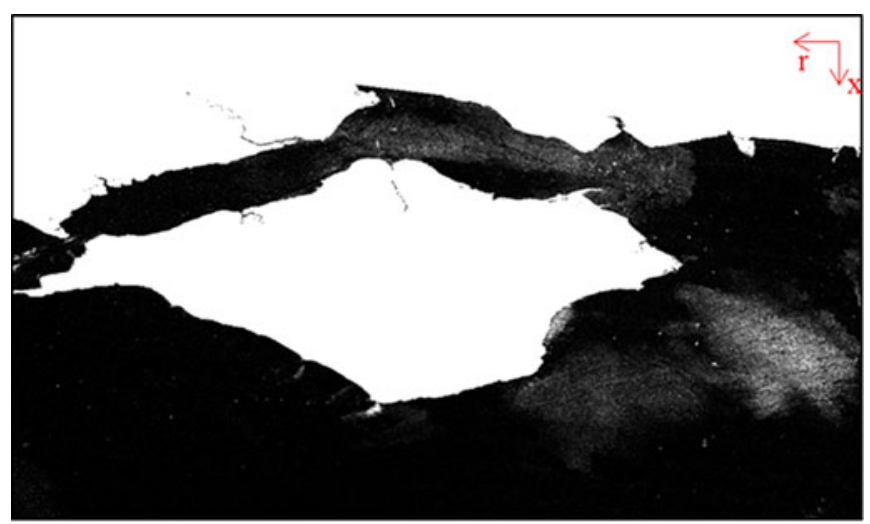

(a)

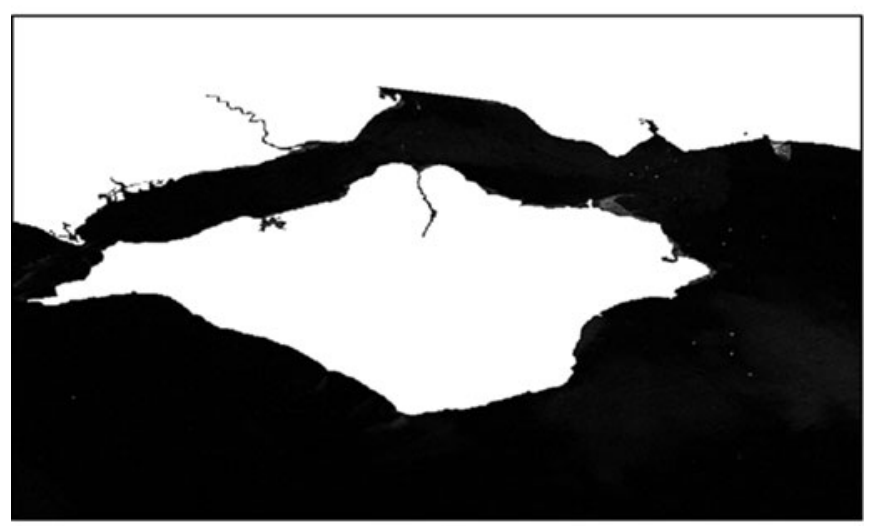

(b)

Fig. 11. SAR intensity image acquired from the TerraSAR-X sensor in VV polarization and $\mathrm{X}$ band on 07/06/2016 in ground range $(r)$ /azimuth $(x)$ plane (a). GLRT SAR image (b).

Conversely, the computational load required by the CFAR is lighter than the GLRT because the CFAR does not need to estimate the target parameters and it results in a faster detection process. Indeed, the CFAR is now only 1.5 times faster than the GLRT, taking $26.26 \mathrm{~s}$ to process the over 15 million pixels against the $48.3 \mathrm{~s}$ of the GLRT. The computational time is evaluated by using the same processor described in the simulations of Section IV.

The second dataset analyzed was acquired by the TerraSAR-X sensor on 7th June, 2016 with a multilook ground range detected (MGD) ScanSAR format with VV polarization and a spatial resolution of $16.5 \mathrm{~m}$ in both azimuth and ground range direction. Here, the clutter presents a much higher intensity on average (around $7 \mathrm{~dB}$ ) compared to the previous image. The intensity SAR images (where the land pixels are masked out) before and after applying the GLRT are shown in Fig. 11(a) and (b), re-
TABLE VI

CFAR AND GLRT PERFORMANCE COMPUTED ON TERRASAR B DATASET

\begin{tabular}{lcc}
\hline \hline & CFAR & GLRT \\
\hline TCR [dB] & 19.4 & 41.1 \\
AIS detected & 6 & 6 \\
False alarm detected & 21 & 1 \\
Total targets detected & 25 & 28 \\
Computational time [s] & 55.4 & 70.5 \\
\hline \hline
\end{tabular}

spectively. It is clear from a visual inspection that the clutter is not homogeneous with a stronger and spiky backscattering on the eastside of the Isle of Wight. This area (worst-case scenario for the ship detection algorithm) has been chosen to statistically characterize the sea clutter. As expected, the Gaussian model (whose multilooked intensity is Gamma distributed [33]) is not valid anymore and the $K$-distribution is the best statistical model to represent the sea backscattering [13], [35]. After computing the clutter and the target parameters, the GLRT function [see (3)] is derived and the enhanced SAR image is finally shown in Fig. 11(b). Again, the novel algorithm works also in presence of stronger clutter: the sea surface appears much darker in this image than in the original SAR image and the TCR is substantially higher. Main results for both CFAR and GLRT are summarized in Table VI. Similarly to the previous dataset, the GLRT works with an enhanced TCR with an increment of $21.7 \mathrm{~dB}$ slightly lower than the TCR increment evaluated in the homogeneous clutter datasets and, as a consequence, it is able to detect more targets (28 against 25) than the CFAR algorithm. In addition, it is much more robust to the spiky behavior of the sea surface caused by the heavy tail of the clutter distribution. Indeed, only a single false alarm is detected with the GLRT compared to the 21 detected using the CFAR. It is important to underline that fewer false alarms are detected if a clustering phase is implemented as done in [25] and [45] or if an adaptive threshold algorithm (using a moving window for the clutter and targets parameters evaluation) is chosen at the cost of a heavier computational load. Finally, as expected from the analysis of all the other images, the computational time of the GLRT is higher than that of the CFAR.

\section{B. Sentinel-1 Dataset}

The third image is a ground range detected (GRD) dataset acquired from the Sentinel-1 sensor at C-band over the Portsmouth harbor on December 1st, 2014. The SAR image is multilooked $(6 \times 6$ equivalent to $34.4 \mathrm{ENL})$ to achieve a spatial resolution of $23 \mathrm{~m}$ in both azimuth and ground range.

The calibration and the land masking are both performed by using the Sentinel-1 tool for SAR images [44]. In Fig. 12(a), the intensity of the SAR image is shown, while in Fig. 12(b), the masking outcomes are displayed. White pixels always represent the land and the black ones the sea clutter.

Dealing with level-1 GRD images from Sentinel-1, the sea clutter can be modeled with a Gaussian distribution, while the L-look intensity is Gamma distributed [33]. The canonical target is modeled within the GO-GO approximation shown in Fig. 6 . 


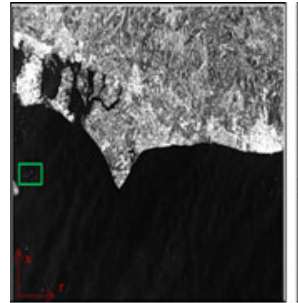

(a)

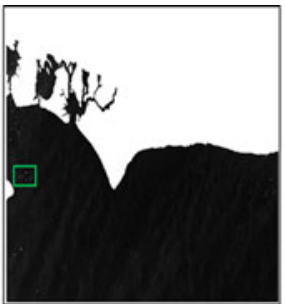

(b)

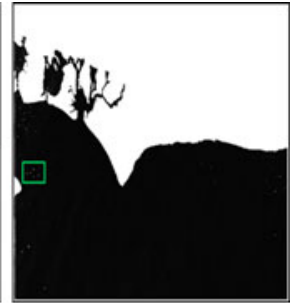

(c)
Fig. 12. SAR intensity image from the Sentinel-1 dataset acquired in HH polarization and $\mathrm{C}$ band in ground range ( $r$ )/azimuth $(x)$ plane (a). Land masking outcomes (b). GLRT SAR image (c). The green rectangle is the ROI isolated to analyzed the TCR.
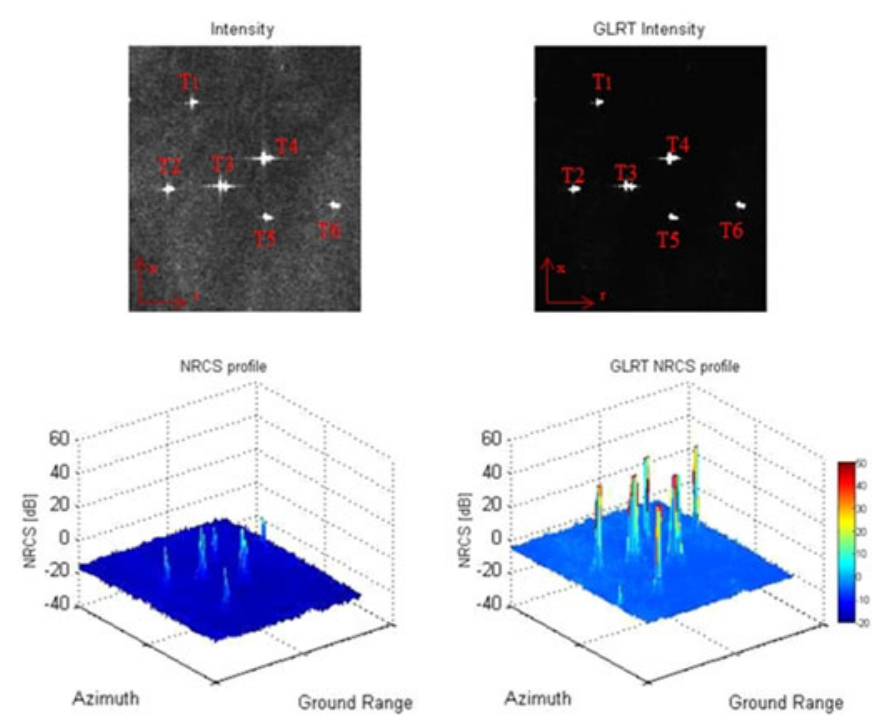

Fig. 13. ROI Intensity image from Sentinel sensor before and after applying the generalized-likelihood function band in ground range $(r)$ /azimuth $(x)$ plane at the top. $T_{1}, T_{2}, T_{3}, T_{4}, T_{5}$, and $T_{6}$ are the targets signatures. NRCS profile relative to the ROI before and after applying the generalized-likelihood function at the bottom.

Both clutter and target parameters have been computed using the MATLAB MLE function similarly to what has been done for the TerraSAR-X dataset. The ENL, instead, has been retrieved from the ancillary data of the sensor for this GRD product.

The results of the GLRT algorithm are finally displayed in Fig. 12(c). In order to evaluate the improvement in the TCR, a ROI of $400 \times 400$ pixels is selected (see the green box in Fig. 12), which includes the signatures of six (out of eight) AIS signals. At the top panel of Fig. 13, the ROI of the intensity image is shown before and after applying the generalized-likelihood function, while the relative NRCS profile in decibel is shown at the bottom panel of Fig. 13. It has been calculated that the TCR improves from 18.92 to $48.96 \mathrm{~dB}$ with an increment of more than $30 \mathrm{~dB}$ [45].

As for the TerraSAR-X dataset, a PFA of $10^{-7}$ with a Global Threshold is used to evaluate the GLRT and CFAR algorithms. Results are reported in Table VII in terms of TCR, objects detected, and computational time.

The azimuth ambiguities are removed from the detected objects by evaluating the theoretical displacement between the
TABLE VII

CFAR AND GLRT PERFormanCE COMPUTED ON SENTINEL-1 DATASET

\begin{tabular}{lcc}
\hline \hline & CFAR & GLRT \\
\hline TCR [dB] & 18.9 & 48.9 \\
AIS detected & 8 & 8 \\
Total targets detected & 21 & 26 \\
Computational time [s] & 26.3 & 40.9 \\
\hline \hline
\end{tabular}

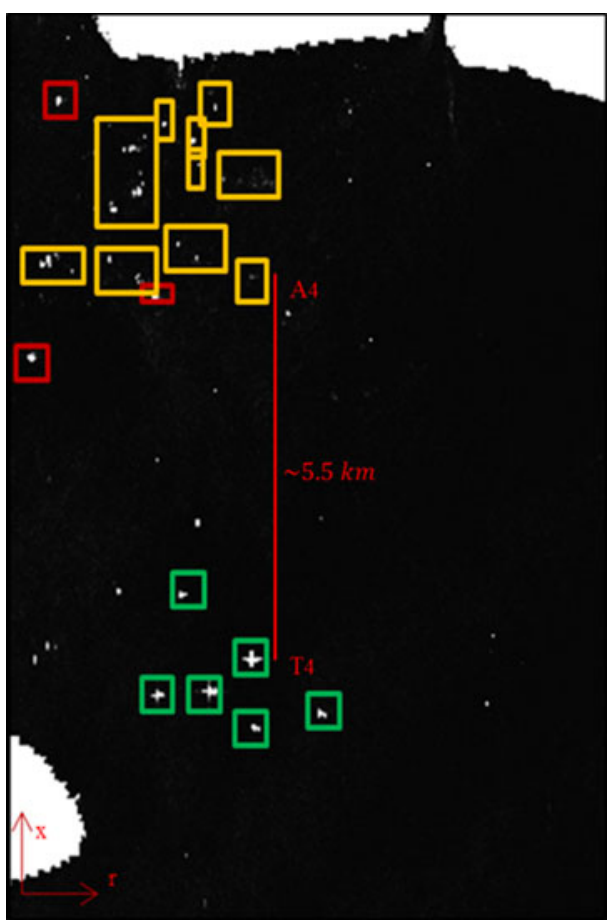

Fig. 14. GLRT SAR image in proximity of the Portsmouth coast. Available AIS signals (green rectangles), azimuth ambiguities (yellow rectangles), cylindrical forts (red rectangles). $T_{4}$ and $A_{4}$ are a ship target and its first order replica, respectively.

target and its first order replica, as already performed in [3] and [26]. The azimuth distance between the target and the first order replicas is given by the following [3]:

$$
\Delta x_{a z}=\frac{\lambda R}{2 V} \operatorname{prf}
$$

where $\Delta x_{a z}$ is the azimuth displacement, $R$ is the range distance between the target and the sensor, $\lambda$ is the wavelength, prf is the pulse repetition frequency, and $V$ is the sensor velocity. From the ancillary data, it has been retrieved that $\lambda=0.06 \mathrm{~m}, R=$ $856 \times 10^{3} \mathrm{~m}$, prf $=1.65 \mathrm{kHz}$, and $V=7.7 \times 10^{3} \mathrm{~m} / \mathrm{s}$ and, consequently, $\Delta x_{a z}=5.5 \times 10^{3} \mathrm{~m}$. However, more efficient techniques exist in the literature to reject azimuth ambiguities based on the Wiener selective filter [46], [47], which can be used to better clean the SAR images.

A zoom-in GLRT SAR of the Portsmouth coast is shown in Fig. 14 where most of the azimuth ambiguities are visible. Azimuth ambiguities are mainly located in this area due to the proximity of several tall buildings and skyscrapers (spinnaker tower and the university campus). In addition, the replicas relative to some strong scatterers over the sea (tankers and cargos) 


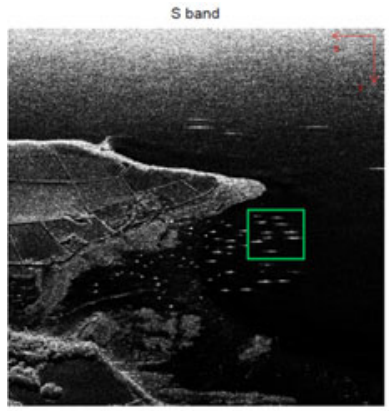

(a)

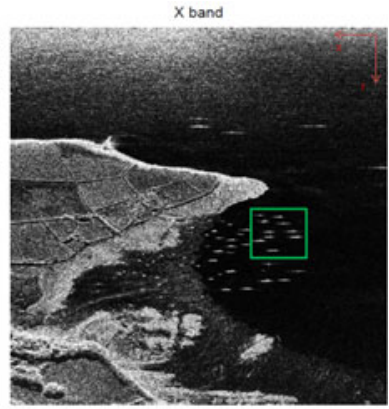

(b)
Fig. 15. SAR intensity images from the Airbus airborne demonstrator acquired in $\mathrm{HH}$ polarization in slant range $(r)$ /azimuth $(x)$ plane at $\mathrm{S}$ band (a) and $\mathrm{X}$ band (b). The green rectangle is the ROI isolated to analzse the TCR.

are also visible. The first-order replica $\left(A_{4}\right)$, relative to the target $T_{4}$ of Fig. 13 is highlighted in Fig. 14. In addition, the ship signatures with available AIS data, the azimuth ambiguities, and three cylindrical forts situated in the area are properly highlighted in Fig. 14 as green, yellow, and red boxes, respectively. These forts are found on the SAR image by matching their latitude and longitude on atlas reporting the position of forts and buoys in the Solent area (available at [48]). The same forts could be rejected and included in the land mask if DEM data with a higher resolution were available. Azimuth replicas and fort signatures are not included in the detection analysis and in the results reported in Table VII. All the other bright spots in the SAR images of Fig. 13 can be regarded as potentially genuine targets.

The GLRT and CFAR algorithms have correctly detected the eight AIS signals available. However, the GLRT is able to detect more targets (26 against 21 retrieved by CFAR) since it provides a higher TCR (48.96 dB against $18.92 \mathrm{~dB}$ ). Conversely its computational load is heavier than CFAR one (40.85 against $26.26 \mathrm{~s})[45]$.

\section{Airbus Dataset}

The last dataset was acquired from the Airbus airborne demonstrator over the Angle Bay in the Pembrokeshire in U.K. on July 28th, 2010. The airborne sensor acquired a dataset both at $\mathrm{S}$ - and X-bands simultaneously. This dataset is meaningful because it allows us to compare directly the detection performance at two different bands acquired with the same clutter and kind of targets. Unfortunately, the ground truth is not available and no AIS signal is retrieved for this dataset. In addition, since it is an airborne acquisition, the radar look angle greatly varies between the near and far range (with an excursion of $37^{\circ}$ ) as reported in the parameters acquisition in Table IV.

Before processing the images (at $\mathrm{S}$ - and X-bands), the absolute calibration is performed as described in [49] by using a square trihedral reflector with a 0.80-m side. In Fig. 15(a) and (b), the intensity SAR image is shown at S- and X-bands, respectively. It is clear that the NRCS relative to the sea clutter is much higher in proximity of the near range and it decreases with the increasing of the range distance, as shown in Fig. 16. The latter shows the NRCS profile of the sea clutter against the variation of the radar look angle $\vartheta$ at both bands. Most of the

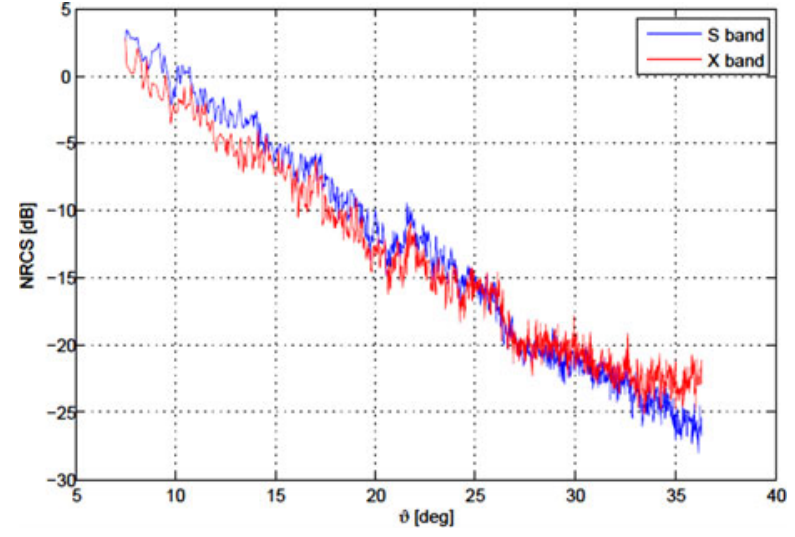

Fig. 16. NRCS plot relative to the sea clutter against the radar look angle ( $\vartheta$ ) for a cut at constant azimuth at $\mathrm{X}$ band (red line) and $\mathrm{S}$ band (blue line).
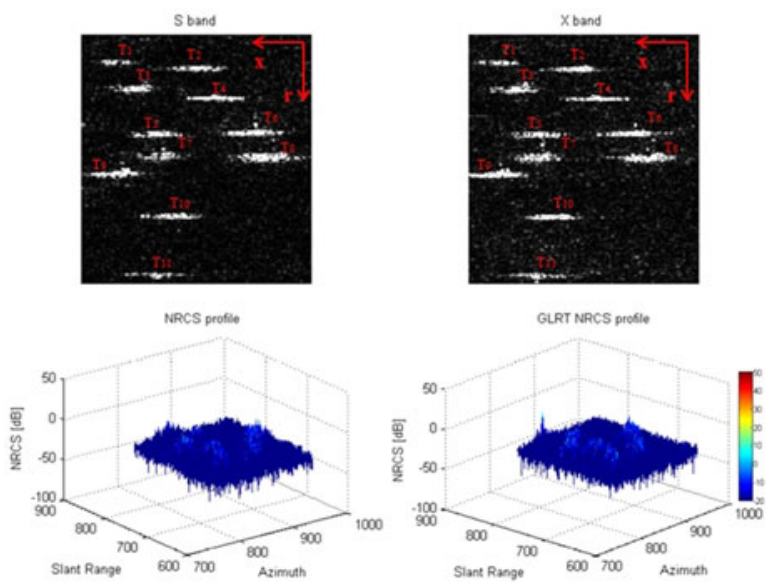

Fig. 17. ROI Intensity image from Airbus demonstrator in slant range (r)/azimuth $(x)$ plane at $\mathrm{X}$ and $\mathrm{S}$ band at the top. $T_{i}$, with $i=1, . .11$, are the targets signatures. NRCS profile relative to the ROI at X and $\mathrm{S}$ band at the bottom.

electromagnetic radiation is backscattered in specular radiation and the SAR pixels located in the near range $\left(\vartheta=7^{\circ}\right)$ present a high NRCS (around $3 \mathrm{~dB}$ for both X- and S-band), thus appearing much brighter than the sea pixels in the far range region (where the NRCS is below $-20 \mathrm{~dB}$ for both X-and S-bands).

From a visual inspection, it is possible to notice a low tide region between the open sea and the land. It is clear that this low tide area is more easily distinguishable at X-band; targets present in the same region are more easily detectable at S-band. However, a ROI of $200 \times 180$ pixels in azimuth and slant range, respectively, is isolated in the open sea area for ship-detection purposes. The ROI is highlighted with a green rectangle in Fig. 15 and presents an average look angle of $30^{\circ}$, typical of most spaceborne SAR acquisitions. In Fig. 17, the ROI intensities including the signatures of 11 ships and their relative NRCS profiles are shown at $\mathrm{S}$ - and X-bands. It results that TCR is $17.5 \mathrm{~dB}$ at S-band and 18.1 at X-band by applying (12).

In order to apply the generalized-likelihood function $\Lambda_{G}(\cdot)$, an exponentially distributed clutter has been assumed for the clutter intensity. The GLRT SAR intensities relative to the ROI and the corresponding NRCS profile are displayed in Fig. 18 at 


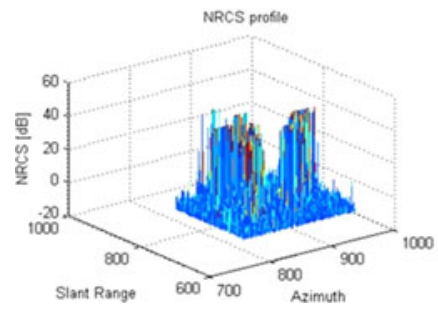

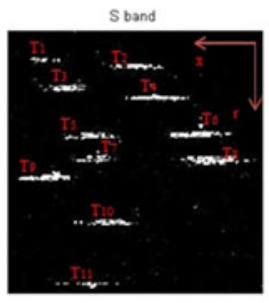
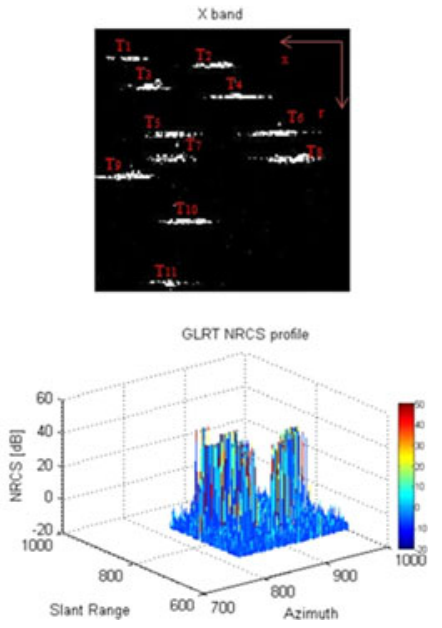

Fig. 18. GLRT ROI intensity image from Airbus demonstrator in slant range $(r) /$ azimuth plane at $\mathrm{X}$ and $\mathrm{S}$ band at the top. $T_{i}$, with $i=1, . .11$, are the targets signatures. NRCS profile relative to the GLRT ROI at $\mathrm{X}$ and $\mathrm{S}$ band at the bottom.

TABLE VIII

CFAR AND GLRT PERFORMANCE COMPUTED ON AIRBUS DEMONSTRATOR DATASET

\begin{tabular}{lccccc}
\hline \hline & \multicolumn{2}{c}{$\mathrm{S}$ band } & & \multicolumn{2}{c}{$\mathrm{X}$ band } \\
\cline { 2 - 3 } \cline { 5 - 6 } \cline { 5 - 6 } & CFAR & GLRT & & CFAR & GLRT \\
\hline TCR [dB] & 17.5 & 50.1 & & 18.1 & 50.4 \\
AIS detected & $N a$ & $N a$ & & $N a$ & $N a$ \\
Total targets detected & 11 & 11 & & 11 & 11 \\
Computational time [s] & 0.6 & 1.9 & & 0.6 & 1.9 \\
\hline \hline
\end{tabular}

$\mathrm{S}$ - and X-bands. It results that the TCR is greatly improved (50.1 and $50.4 \mathrm{~dB}$ at $\mathrm{S}$ - and X-bands, respectively) after applying the function $\Lambda_{G}(\cdot)$. Outcomes for both bands and algorithms (CFAR and GLRT) are finally summarized in Table VIII where a PFA $=10^{-7}$ with a global threshold is used. Both CFAR and GLRT are able to detect the 11 targets included in the ROI at both bands. However, as already underlined in the analyses of Figs. 17 and 18, the GLRT exhibits a much higher TCR (more than $32 \mathrm{~dB}$ at both bands) although its computational time is more than three times slower.

\section{CONCLUSION}

A novel GLRT-based technique for ship detection in SAR imagery has been introduced. Differently from the traditional CFAR algorithm, the GLRT approach is based also on the target distribution, which is modeled according to the GO model already presented in [25] by Iervolino et al.

The theoretical TCR has been evaluated at $\mathrm{HH}$ polarization for S-, C-, and X-bands. Results show only a slight difference in the TCR with the band variation (see Fig. 3). As a consequence, the detector performance is similar at different bands as it has been demonstrated through the Monte-Carlo simulations. ROC curves, derived from SAR simulations, show that GLRT performs better than CFAR (higher PD for any fixed PFA) at every band (see Fig. 6). Conversely, the CFAR results more than three times faster than the GLRT because it requires fewer steps to be processed. Indeed, the MLE estimation of target parameters is not required for the CFAR. However, both algorithms can be used in RT or NRT applications where the number of pixels to be analyzed is in the order of tens or hundreds millions.

Other simulations have demonstrated that the GLRT performs better for $\sigma_{\mathrm{dev}} / L=0.2$ and $\vartheta=40^{\circ}$ (see Fig. 7). In addition, it is advisable to perform a multilooking operation if a highresolution SAR image is available. Indeed, it has been shown that the detector's performance greatly improves with the increase of ENL although the spatial resolution is reduced by a factor $1 / \mathrm{ENL}$.

The GLRT technique has finally been tested and compared to the CFAR on four different real datasets acquired from TerraSAR-X (at X-band), Sentinel-1 (at C-band), and the Airbus airborne demonstrator (at $\mathrm{S}$ - and $\mathrm{X}$-bands). Outcomes show that the GLRT is able to detect more targets (see Tables V-VIII) when the PFA is fixed and present a much higher TCR (between 22 and $32 \mathrm{~dB}$ on average). However, the propose approach is slightly slower as already expected from the simulations results.

The two main findings of this paper can be summarized as follows.

1) Based on the available real SAR datasets and following the removal of azimuth ambiguities, it results that the GLRT method works effectively for ship detection purposes, showing very good and similar performance for all the considered bands. This suggests that multisource SAR images (acquired from different sensors at different bands and polarizations) can in principle be conjunctly used to constantly monitor sea areas and harbors of particularx interest.

2) By processing both SAR simulated and real datasets, it has been demonstrated that the GLRT provides benefits compared to the traditional global CFAR algorithm. These benefits are evident when the detection is more challenging (low TCR and heterogeneous clutter). Conversely, the CFAR is a faster algorithm and provides close performance in case of high TCR as shown for the AIS signatures detected by both approaches for the TerraSAR-X and Sentinel-1 datasets. The GLRT should, therefore, be used in the area of low TCR, where it is able to detect more targets than the CFAR algorithm.

The authors are currently working on the inclusion of some efficient techniques for the azimuth ambiguities removal in the detection chain and the improvement of the target model. Future outlooks would include the analytical evaluation of the detector performance (in terms of PFA and PD) if a close solution is found for the $\Lambda_{G}$ pdf given the hypotheses $H_{0}$ and $H_{1}$. Furthermore, a comparison between the GLRT algorithm with sublooks and improved CFAR detectors over more datasets will be conceived to get a complete picture of ship detectors performance in SAR imagery.

\section{ACKNOWLEDGMENT}

The authors would like to thank the German Aerospace Centre for providing the TerraSAR-X dataset within the COA1857 proposal and the SAR multifrequency ship-detection project, the 
European Space Agency for the Sentinel-1 dataset, and Airbus UK for the airborne SAR data.

\section{REFERENCES}

[1] (2015, Jun. 9). European Maritime Safety Agency (EMSA): CleanSeaNet. [Online]. Available: http://www.emsa.europa.eu/csn-menu.html

[2] (2015, Jun. 9). European Maritime Safety Agency (EMSA): Vessel Traffic Monitoring in EU Waters (SafeSeaNet). [Online]. Available: http://www.emsa.europa.eu/ssn-main.html

[3] S. Brusch, S. Lehner, T. Fritz, and M. Soccorsi, "Ship surveillance with TerraSAR-X," IEEE Trans. Geosci. Remote Sens., vol. 49, no. 3, pp. 1092-1103, Mar. 2011.

[4] (2015, Jun. 9). International Maritime Organization (IMO): LongRange Identification and Tracking (LRIT). [Online]. Available: http://www.imo.org/OurWork/Safety/Navigation/Pages/LRIT.aspx

[5] A. Hillman et al., "RADARSAT-2 initial system operations and performance," in Proc. Int. Geosci. Remote Sens. Symp., Cape Town, South Africa, Jul. 2009, pp. 753-756.

[6] (2013, May 15). e-Geos (2013) COSMO-SkyMed System Description and User Guide. [Online]. Available: http://www.e-geos.it/

[7] J. Louet and S. Bruzzi, "ENVISAT mission and system," in Proc. Int Geosci. Remote Sens. Symp., Hamburg, Germany, Jul. 1999, pp. 16801682.

[8] A. Panetti et al., "Sentinel-1 spacecraft," in Proc. Eur. Conf. Synthetic Aperture Radar, Berlin, Germany, Jun. 2014, pp. 4-7.

[9] G. Krieger, A. Moreira, H. Fiedler, I. Hajnsek, M. Weerner, and M. Younis, "TanDEM-X: A satellite formation for high-resolution SAR interferometry," IEEE Trans. Geosci. Remote Sens., vol. 45, no. 11, pp. 3317-3341, Nov. 2007.

[10] Y. Arikawa, T. Yamamoto, Y. Kondoh, and K. Akiyama, "ALOS-2 orbit control and determination," in Proc. Int. Geosci. Remote Sens. Symp., Quebec, Canada, Jul. 2014, pp. 3415-3417.

[11] R. Bird, P. Whittaker, B. Stern, N. Angli, M. Cohen, and R. Guida, "NovaSAR-S: A low cost approach to SAR applications," in Proc. Asia-Pac. Conf. Synthetic Aperture Radar, Tsukuba, Japan, Sep. 2013, pp. 84-87.

[12] R. Solorza, C. Notarnicola, and H. Karszenbaum, "Retrieval of soil moisture using electromagnetic models and a Bayesian approach in view of the SAOCOM mission: Study on SARAT images in an agricultural site in Argentina," in Proc. Int. Geosci. Remote Sens. Symp., Melbourne, Australia, Jul. 2013, pp. 739-742.

[13] D. J. Crisp, "The state-of-art in ship detection in synthetic aperture radar imagery," DSTO Information Science Lab., Canberra, Australia, Tech. Rep. DSTO-RR-0272, 2004.

[14] Y. Cui, J. Yang, Y. Yamaguchi, G. Singh, S. Park, and H. Kobayashi, "On semiparametric clutter estimation for ship detection in synthetic aperture radar images," IEEE Trans. Geosci. Remote Sens., vol. 51, no. 5, pp. 3170-3180, May 2013.

[15] X. Leng, K. Ji, K. Yang, and H. Zou, "A bilateral CFAR algorithm for ship detection in SAR images," IEEE Geosci. Remote Sens. Lett., vol. 12, no. 7, pp. 1536-1540, Jul. 2015.

[16] J.-C. Souyris, C. Henry, and F. Adragna, "On the use of complex SAR image spectral analysis for target detection: Assessment of polarimetry," IEEE Trans. Geosci. Remote Sens., vol. 41, no. 12, pp. 2725-2734, Dec. 2003

[17] A. Marino, M. Sanjuan-Ferrer, I. Hajnsek, and K. Ouchi, "Ship detection with analysis of synthetic aperture radar: A comparison of new and wellknown algorithms," Remote Sens., vol. 7, no. 5, pp. 5416-5439, 2015.

[18] C. Brekke, S. Normann Anfinsen, and Y. Larsen, "Subband extraction strategies in ship detection with the subaperture cross-correlation magnitude," IEEE Geosci. Remote Sens. Lett., vol. 10, no. 4, pp. 786-790, Jul. 2013.

[19] R. Z. Schneider, K. P. Papathanassiou, I. Hajnsek, and A. Moreira, "Polarimetric and interferometric characterization of coherent scatterers in urban areas," IEEE Trans. Geosci. Remote Sens., vol. 44, no. 4, pp. 971-984, Apr. 2006

[20] A. Marino, "A notch filter for ship detection with polarimetric SAR data," IEEE J. Sel. Topics Appl. Earth Obs. Remote Sens., vol. 6, no. 3, pp. 1219-1232, Mar. 2013.

[21] D. Tao, C. Brekke, and S. Anfinsen, "An experimental study on ship detection based on the fixed-point polarimetric whitening filter," Proc. SPIE, vol. 8180, Oct. 2011, Art. no. 81800Y.

[22] A. Marino and I. Hajnsek, "Statistical test for a ship detector based on the polarimetric notch filter," IEEE Trans. Geosci. Remote Sens., vol. 53, no. 8, pp. 4578-4595, Aug. 2015.
[23] M. Rey, "Results from the crusade ship detection trial: Polarimetric SAR," in Proc. Int. Geosci. Remote Sens. Symp., Toronto, Canada, 2002, vol. 2, pp. 711-713.

[24] C. Liu, P. Vachon, and G. Geling, "Improved ship detection using polarimetric SAR data," in Proc. Int. Geosci. Remote Sens. Symp., Anchorage, AK, USA, 2004, vol. 3, pp. 1800-1803.

[25] P. Iervolino, R. Guida, and P. Whittaker, "A model for the backscattering from a canonical ship in SAR imagery," IEEE J. Sel. Topics Appl. Earth Obs. Remote Sens., vol. 9, no. 3, pp. 1163-1175, Mar. 2016.

[26] P. Iervolino, R. Guida, and P. Whittaker, "NovaSAR-S and maritime surveillance," in Proc. Int. Geosci. Remote Sens. Symp., Melbourne, Australia, Jul. 2013, pp. 1282-1285.

[27] M. Sciotti, D. Pastina, and P. Lombardo, "Polarimetric detectors of extended targets for ship detection in SAR images," in Proc. Int. Geosci. Remote Sens. Symp., Sydney, Australia, 2001, pp. 3132-3134.

[28] S. Kay, Fundamentals of Statistical Signal Processing, vol. 2. Upper Saddle River, NJ, USA: Prentice-Hall, 2011.

[29] A. De Maio, G. Fornaro, and A. Pauciullo, "Detection of single scatterers in multidimensional SAR imaging," IEEE Trans. Geosci. Remote Sens., vol. 47, no. 7, pp. 2284-2297, Jul. 2009.

[30] E. Conte, M. Lops, and G. Ricci, "Asympotically optimum radar detection in compound-Gaussian clutter," IEEE Trans. Aerosp. Electron. Syst., vol. 31, no. 2, pp. 617-625, Feb. 1995.

[31] G. Franceschetti, A. Iodice, and D. Riccio, "A canonical problem in electromagnetic backscattering from buildings," IEEE Trans. Geosci. Remote Sens., vol. 40, no. 8, pp. 1787-1801, Aug. 2002.

[32] R. Guida, A. Iodice, and D. Riccio, "Height retrieval of isolated buildings from single high-resolution SAR Images," IEEE Trans. Geosci. Remote Sens., vol. 48, no. 7, pp. 2967-2979, Aug. 2010.

[33] J. Lee and E. Pottier, Polarimetric Radar Imaging: From Basics to Applications. Boca Raton, FL, USA: CRC Press, Taylor \& Francis, 2009.

[34] P. Iervolino, R. Guida, and P. Whittaker, "Roughness parameters estimation of sea surface from SAR images," in Proc. Int. Geosci. Remote Sens. Symp., Quebec, Canada, Jul. 2014, pp. 5013-5016.

[35] K. Ward, R. Tough, and S. Watts, Sea Clutter: Scattering, the K Distribution and Radar Performance, 2nd ed. London, U.K.: The Institution of Engineering and Technology, 2013.

[36] F. Ulaby and D. Long, Microwave Radar and Radiometric Remote Sensing. Ann Arbor, MI, USA: Univ. of Michigan Press, 2013.

[37] J. Wei, J. Zhang, G. Huang, and Z. Zhao, "On the use of cross-correlation between Volume scattering and helix scattering from polarimteric SAR data for the improvement of ship detection," Remote Sens., vol. 8, no. 1, pp. 1-16, 2016.

[38] R. Guida, A. Iodice, D. Riccio, and U. Stilla, "Model-based interpretation of high-resolution SAR images of buildings," IEEE J. Sel. Topics Appl. Earth Obs. Remote Sens., vol. 1, no. 2, pp. 107-119, Jun. 2008

[39] (2015, Jun. 9). National Centre for Environment Information (NOAA): World Ocean Atlas 2009 Figures (WOA09F). [Online]. Available: https://www.nodc.noaa.gov/OC5/WOA09F/pr_woa09f.html

[40] (2015, Jun. 9). DLR: Radiometric Calibration of TerraSAR-X Data. [Online]. Available: http://www2.geo-airbusds.com/files/pmedia/public/ r465_9_tsx-x-itd-tn-0049-radiometric_calculations_i3.00.pdf

[41] R. B. D'Agostino and M. A. Stephens, Goodness of Fit Techniques. New York, NY, USA: Marcel Dekker, 1986.

[42] P. Iervolino, R. Guida, and P. Whittaker, "A new GLRT-based ship detection technique in SAR images," in Proc. IEEE Int. Geosci. Remote Sens. Symp., Milan, Italy, Jul. 2015, pp. 3131-3134.

[43] (2015, Jun. 9). AIS Validation Data. [Online]. Available: http://www. shipais.com

[44] (2015, Jun. 2). European Space Agency. Sentinel-1 User Handbook. [Online]. Available: https://sentinel.esa.int/

[45] P. Iervolino, R. Guida, and P. Whittaker, "A novel ship-detection technique for sentinel-1 SAR data," in Proc. 2015 IEEE 5th Asia-Pac. Conf. Synthetic Aperture Radar, Singapore, Sep. 2015, pp. 797-801.

[46] G. Di Martino, A. Iodice, D. Riccio, and G. Ruello, "Filtering of azimuth ambiguity in stripmap synthetic aperture radar images," IEEE J. Sel. Topics Appl. Earth Obs. Remote Sens., vol. 7, no. 9, pp. 3967-3978, Sep. 2014.

[47] A. Monti Guarnieri, "Adaptive removal of azimuth ambiguities in SAR images," IEEE Trans. Geosci. Remote Sens., vol. 43, no. 3, pp. 625-633, Mar. 2005.

[48] (2015, Jun. 2). Royal Southern Yacht Club. Solent Buoys. [Online]. Available: http://www.royal-southern.co.uk/Afloat/Solent-Buoys

[49] A. Freeman, "Radiometric calibration of SAR image data," in Proc. XVII Congr. Int. Soc. Photogramm. Remote Sens., 1992, pp. 212-222. 


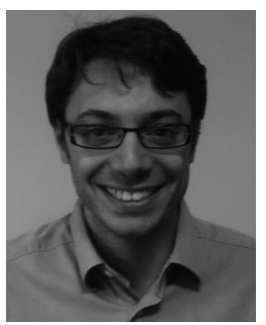

Pasquale Iervolino was born in Naples, Italy, on August 16, 1985. He received the B.S. (cum laude) and M.S. (cum laude) degrees in telecommunications engineering from the University of Naples Federico II, Naples, Italy, in 2008 and 2010, respectively, the Ph.D. degree in electronic engineering from the Surrey Space Centre, University of Surrey, Guildford, U.K., in 2016.

Since 2015, he has been working as a Research Fellow at the Surrey Space Centre on several remote sensing projects. His research interests include microwave remote sensing, the development and inversion of scattering models from natural and man-made surfaces, and synthetic aperture radar ship-detection algorithms.

Dr. Iervolino received the Student Paper Award published on the IEEE Proceedings of the 2015 Asia-Pacific Conference on Synthetic Aperture Radar (APSAR).

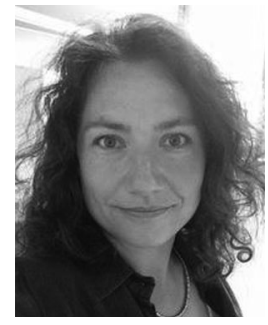

Raffaella Guida (S'04-M'08) was born in Naples, Italy, on October 24, 1975. She received the Laurea degree (cum laude) in telecommunications engineering and the Ph.D. degree in electronic and telecommunications engineering from the University of Naples Federico II, Naples, Italy, in 2003 and 2007, respectively.

In 2006, she received a two-year research grant from the University of Naples Federico II to be spent at the Department of Electronic and Telecommunication Engineering on the topic of electromagnetic field propagation in urban environment. In 2006, she was also a Guest Scientist with the Department of Photogrammetry and Remote Sensing, Technische Universität München, Munich, Germany. In 2008, she joined, as a Lecturer of satellite remote sensing, the Surrey Space Centre (SSC), University of Surrey, Guildford, U.K., where she is currently a Senior Lecturer and leads the Remote Sensing Applications research group. Her main research interests include electromagnetics and microwave remote sensing, particularly in simulation and modeling of synthetic aperture radar signals relevant to natural surfaces and urban scenes, new remote sensing mission concepts, and applications.

Dr. Guida received the IEEE J-STARS Best Paper Award 2013. 